\title{
Large-scale allosteric switch in the 7SK RNA regulates transcription in response to growth and stress
}

Samuel W. Olson ${ }^{1}$, Anne-Marie W. Turner ${ }^{2,3}$, J. Winston Arney ${ }^{1}$, Irfana Saleem ${ }^{4}$, Chase A.

Weidmann $^{1}$, David M. Margolis ${ }^{2,3}$, Kevin M. Weeks ${ }^{1,7, *}$ and Anthony M. Mustoe ${ }^{4,5, *}$

${ }^{1}$ Department of Chemistry, University of North Carolina, Chapel Hill, NC 27599-3290

${ }^{2}$ Department of Medicine, University of North Carolina, Chapel Hill, NC 27599

${ }^{3}$ UNC HIV Cure Center, University of North Carolina, Chapel Hill, NC 27599

${ }^{4}$ Verna and Marrs McClean Department of Biochemistry and Molecular Biology; Therapeutic Innovation Center (THINC), Baylor College of Medicine, Houston, TX, 77030

${ }^{5}$ Department of Molecular and Human Genetics; Baylor College of Medicine, Houston, TX, 77030

*correspondence, weeks@unc.edu and anthony.mustoe@bcm.edu

${ }^{7}$ Lead contact 


\section{Summary}

7SK is a highly conserved non-coding RNA that regulates eukaryotic transcription by sequestering positive transcription elongation factor $\mathrm{b}(\mathrm{P}-\mathrm{TEFb})$. 7SK regulatory function likely entails changes in RNA structure, but characterizing dynamic RNA-protein complexes in cells has remained an unsolved challenge. We describe a new chemical probing strategy (DANCE$\mathrm{MaP}$ ) that uses maximum likelihood deconvolution and probabilistic read assignment to define simultaneously (i) per-nucleotide reactivity profiles, (ii) direct base pairing interactions, and (iii) tertiary and higher-order interactions for each conformation of multi-state RNA structural ensembles, all from a single experiment. We show that human 7SK RNA, despite significant heterogeneity, intrinsically codes for a large-scale structural switch that couples dissolution of the P-TEFb binding site to structural remodeling at distal release factor binding sites. The 7SK structural equilibrium is regulated by cell type, shifts dynamically in response to cell growth and stress, and can be exogenously targeted to modulate transcription in cells. Our data support that the 7SK structural ensemble functions as an integrator of diverse cellular signals to control transcription elongation in environment and cell specific ways, and establishes DANCE-MaP as a powerful strategy for comprehensively defining RNA structure and dynamics in cells.

\section{Introduction}

RNA molecules fold back on themselves into complex secondary and tertiary structures that provide the basis of specific protein recognition, ligand binding, and broad gene regulatory functions (Sharp, 2009; Cech and Steitz, 2014). Most RNA elements can additionally fold into more than one structure, which can enable RNAs to function as regulatory switches (Dethoff et al., 2012). mRNA-based switches can regulate transcription, splicing, and translation of specific genes in response to metabolites (riboswitches) (Breaker, 2012) and protein binding (Ray et al., 2009; Fu et al., 2013). Large-scale RNA structural dynamics also underpin function of ribonucleoprotein (RNP) complexes such as the ribosome (Rodnina et al., 2017; Sengupta et al., 2019) and the spliceosome (Wilkinson et al., 2020). Nevertheless, despite their broad importance, RNA switches remain exceedingly difficult to identify, quantify in terms of their structure and in-cell equilibria, or link to functional outcomes.

The 7SK RNA is an abundant 332 nucleotide long non-coding RNA, forms the key architectural component of the 7SK small non-coding RNA-protein complex (snRNP), and serves as a major nexus of transcriptional control (Peterlin et al., 2012; Quaresma et al., 2016). 7SK is canonically thought to function by sequestering and inhibiting Cdk9/Cyclin T1 (together termed positive 
transcription elongation factor b, P-TEFb), a kinase required for phosphorylation and release of RNA polymerase II (Pol II) complexes paused at promoter-proximal regions. The P-TEFb-free form of 7 SK appears to play additional roles in facilitating productive elongation (Peterlin et al., 2012; Quaresma et al., 2016), including modulating splicing (Barboric et al., 2009; Egloff et al., 2017; Ji et al., 2021), and chromatin remodeling (Eilebrecht et al., 2011; Flynn et al., 2016). The diverse functions of the 7SK snRNP are driven by coordinated changes in its bound protein components (Krueger et al., 2010). Methylphosphate capping enzyme (MePCE) (Yang et al., 2019) and La related protein 7 (LaRP7) (Krueger et al., 2008; Eichhorn et al., 2018) stabilize the core of the 7SK snRNP. P-TEFb is sequestered through interactions with the accessory protein dimer hexamethylene bis-acetamide inducible protein 1 or 2 (HEXIM1/2), which binds to the 7SK RNA at a high-affinity stem-loop structure, SL1 (Peterlin and Price, 2006; Czudnochowski et al., 2010; Martinez-Zapien et al., 2016). Under transcription stimulatory conditions, P-TEFb and HEXIM1/2 are liberated from 7 SK by diverse release factors, including the bromodomain protein BRD4, and several helicases (Peterlin et al., 2012; Quaresma et al., 2016). The P-TEFbfree form of $7 \mathrm{SK}$ is in turn bound by heterogenous ribonucleoproteins (hnRNPs) and other proteins that interact with sites at the 3' end the 7SK RNA (Peterlin et al., 2012; Quaresma et al., 2016). Most steps of this remodeling process remain poorly understood. Defining 7SK regulatory mechanisms will both illuminate fundamental aspects of transcriptional control and also inform ongoing efforts to inhibit transcription in disease settings, especially cancer (Olson et al., 2018), and, conversely, to activate transcription as part of "kick-and-kill" HIV cure strategies (Richman et al., 2009; Cary et al., 2016).

The 7SK RNA is highly structured (Wassarman and Steitz, 1991) and several studies support the model that P-TEFb binding and release involves remodeling of 7SK RNA structure (Krueger et al., 2010; Brogie and Price, 2017), or that the 7SK RNA exists in distinct conformations depending which proteins are bound (Krueger et al., 2010; Flynn et al., 2016; Brogie and Price, 2017). Multiple models for the 7SK RNA structure have been proposed (Wassarman and Steitz, 1991; Marz et al., 2009; Brogie and Price, 2017; Luo et al., 2021), but the accuracy of these models, whether they represent distinct co-existing states, and how these states might differentially modulate function remains unknown. To date, 7SK structure has primarily been studied using ensemble-average chemical probing approaches that are poorly suited for identifying coexisting RNA conformations or resolving in-cell structural dynamics. Typical of most non-coding RNAs, 7SK sequences show weak sequence covariation, precluding informative evolutionary analysis (Rivas et al., 2016; Kalvari et al., 2021). The 7SK RNP thus 
encapsulates broad features illustrating how RNA structural complexity endows functional complexity, and how such structural complexity frustrates mechanistic understanding.

Single-molecule chemical probing is emerging as a transformative technology for characterizing RNA structure and dynamics in living cells. The foundational conceptual advance is mutational profiling (MaP) reverse transcription, whereby a polymerase reads through and measures multiple chemical adducts per RNA molecule, recording them as mutations in complementary DNA (Homan et al., 2014). Massively parallel sequencing enables measurement of correlated modification events across hundreds of thousands of molecules, which encode rich information regarding RNA ensemble composition (Homan et al., 2014; Tomezsko et al., 2020; Morandi et al., 2021), and through-space secondary (Krokhotin et al., 2016; Cheng et al., 2017; Mustoe et al., 2019) and tertiary (Homan et al., 2014; Dethoff et al., 2018; Sengupta et al., 2019) structure interactions. However, existing single-molecule analysis frameworks only extract one type of information at a time (ensemble composition, base paring, or tertiary interactions). Ensemble deconvolution strategies permit measurement of multiple co-existing per-nucleotide reactivity profiles, but do not directly measure base pairs. Structures for each ensemble state can only be inferred, and this inference problem becomes increasingly ambiguous for long RNAs, particularly in cells. Conversely, the existence of multiple RNA structural states makes it challenging to assign base pairing and tertiary interactions.

Here we present a maximum likelihood (ML) strategy, DANCE-MaP (deconvolution and annotation of ribonucleic conformational ensembles) that extracts and annotates a large fraction of the total information from a single-molecule chemical probing experiment. DANCE-MaP directly visualizes complex RNA ensembles from MaP probing data, including direct detection of base pairs and tertiary interactions for each sub-state, at nucleotide resolution in a single experiment. We extensively benchmark this strategy in the adenine riboswitch, and our data reveal significant, previously undetected, complexity even within this well-defined RNA structural ensemble. We then apply DANCE-MaP to discover a large-scale, sequence-encoded structural switch in the 7SK RNA. Our structural-switch model rationalizes a large body of prior data and directly links P-TEFb release to concerted remodeling of 7SK structure in a cell-type and environmental specific way. We leverage this information to design a prototype, anti-sense oligonucleotide (ASO) to alter the 7SK ensemble and upregulate transcription in cells. Our work establishes DANCE-MaP as a powerful framework for directly resolving complex ensembles in cells and explains diverse features of 7SK IncRNA biology. 


\section{Results}

\section{Deconvolution of complex RNA ensembles with thermodynamic accuracy}

RNA chemical probing data are conventionally analyzed by averaging across all molecules in a sample, yielding a single per-nucleotide reactivity profile that masks underlying dynamics (Fig. $1 \mathrm{~A}$, bottom). In single-molecule probing experiments, each MaP read represents a structural snapshot of an individual RNA molecule. For an RNA that folds into multiple structures, each structure yields distinct groups of reactive and unreactive nucleotides that are co-modified (or unmodified) in a correlated manner (Homan et al., 2014). We implemented a maximum likelihood (ML) framework that uses a modified Bernoulli mixture model to fit single-molecule reads to multiple reactivity profiles, sequentially increasing the number of fitted states until the optimal solution is identified (Fig. 1A, right; S1). Our ML strategy shares features with an independently described algorithm (Tomezsko et al., 2020), but additionally includes adjustments to handle missing data, improve robustness, and capture information from all four RNA nucleotides. Under idealized scenarios, our ML framework can deconvolute ensembles consisting of up to 5 states with populations $\geq 5 \%$ (Fig. S2). Deconvolution accuracy can break down as ensemble heterogeneity increases to $\geq 4$ highly divergent states, meaning that individual deconvoluted states may still contain residual structural heterogeneity. Overall, reactivity profiles and populations are resolved with mean errors $<1 \%$ for ensembles consisting of distinctive states.

We validated our ML framework using the $V$. vulnificus add adenine riboswitch, which folds into a two-state ensemble consisting of a translation OFF state that occludes the Shine-Dalgarno (SD) sequence, and translation ON state that contains an adenine-binding aptamer (Fig. 1) (Reining et al., 2013). Structure probing experiments were performed on an in vitro transcribed RNA using dimethyl sulfate (DMS) under conditions where the reagent reacts with all four nucleotides (Mustoe et al., 2019). Whereas conventional averaging analysis suggests the riboswitch folds into a single well-defined structure, deconvolution of the single-molecule MaP data yielded two reactivity profiles matching the known translation ON and OFF states at the expected $\sim 40: 60$ populations (Fig. 1B) (Reining et al., 2013). Structure-specific differential reactivities are observed at both $A$ and $C$ residues and at $U$ and $G$ (for example, $U 84$ and G85). These data enabled DMS-directed minimum free energy modeling and calculation of pairing probabilities that recapitulate the expected structures (Fig. 1C, left). Pairing probability analysis 
is a routine part of SHAPE-directed structure modeling and provides a key measure of model uncertainty (Siegfried et al., 2014; Smola et al., 2015a; Weeks, 2021), but until now has been unavailable for DMS data. Newly implemented here, pairing probability analysis reveals notable heterogeneity in the P2alt region in the OFF state, consistent with prior observations (Reining et al., 2013; Tomezsko et al., 2020), and with the potential for extended P1 pairing (P1ext) in the ON state (Fig. 1C, right).

We next examined the ability of $\mathrm{ML}$ deconvolution to measure biologically relevant changes in ensemble state by monitoring add riboswitch switching in response to adenine binding. Both OFF and ON conformations are populated even at saturating adenine ligand, consistent with prior studies (Warhaut et al., 2017; Tomezsko et al., 2020) (Fig. 1D, S3). The OFF state reactivity profiles are essentially identical at all adenine concentrations (Fig. 1E, bottom). By contrast, the $\mathrm{ON}$ state exhibits punctate reactivity changes within the ligand binding pocket, indicating adenine-induced stabilization of the aptamer domain tertiary structure (Fig. 1E, top). The concentration-dependence of these reactivity changes are consistent with the ML-resolved ON state representing a mixture of both ligand-free and ligand-bound states. Notably, the population of the $\mathrm{ON}$ state exactly follows the expected binding isotherm, enabling us to compute a $\mathrm{K}_{d} \approx 2 \mu \mathrm{M}$ in agreement with literature values (Reining et al., 2013) (Fig. 1D). The ability to resolve ligand binding thermodynamics with quantitative accuracy is unique to our study and provides rigorous validation of the precision of our ML deconvolution implementation. We further validated our approach using mutants that perturb the OFF-ON equilibrium. Consistent with other studies (Reining et al., 2013; Warhaut et al., 2017; Tian et al., 2018), destabilizing the P2alt helix via the Muton mutation only modestly shifts the equilibrium towards the ON state (Fig. 1E); our data reveal that this (unexpectedly) modest shift reflects creation of a new misfolded third state (Fig. S4). Destabilizing P2 via the Mutoff mutation (new to this study) switches the equilibrium to $65: 35$ between OFF and a new heterogenous state that is not adenine responsive (Fig. 1D, S4).

Collectively, these data validate the robustness and thermodynamic accuracy of our ML deconvolution framework, confirm that the add riboswitch functions via a conformational selection mechanism (Tian et al., 2018), and reveal unexpected complexity in the riboswitch sequence fitness landscape.

\section{Direct measurement of base pairs and tertiary interactions for individual RNA states}


While ML deconvolution provides critical insight into ensemble composition and populations (Fig. 1, S2-S4), the set of RNA base pairs are not visualized directly and must be inferred via structure modeling. This inference problem is often ambiguous, especially for longer RNAs, where multiple sets of pairings can fit the same data, and for RNA structures measured in cells, where bound proteins complicate data interpretation (Smola et al., 2015b). Per-nucleotide reactivity data also do not report potential tertiary interactions. Single-molecule probing data contain characteristic correlations that directly measure through-space base pairing (PAIR, pairing ascertained from interacting RNA strands) and tertiary interactions (RING, RNA interaction groups) (Fig. 2A) (Homan et al., 2014; Mustoe et al., 2019). We developed a read assignment strategy which, in combination with our ML deconvolution approach, enables simultaneous measurement of per-nucleotide reactivity profiles and state-specific base pairing and tertiary interactions in complex structural ensembles. We term this integrated analysis framework deconvolution and annotation of ribonucleic conformational ensembles measured by mutational profiling, or DANCE-MaP.

Given a set of ML-deconvoluted reactivity profiles, individual single-molecule MaP reads can be assigned via a posterior probability calculation to their most likely parent state. State-specific PAIR and RING correlation analysis can then be performed on the assigned reads (Fig. 2A, S1). We devised a two-part strategy to measure state-specific PAIR and RING correlations and circumvent potential assignment biases (see Methods). Analysis of simulated MaP datasets confirmed that DANCE-MaP enables accurate measurement of state-specific RING and PAIR correlations (Fig. S5).

Without ML-deconvolution, PAIR and RING analyses of adenine riboswitch DMS-MaP datasets yield a dense meshwork of correlations consistent with the underlying structural dynamics, but which are challenging to interpret de novo (Fig. 2B, 2C, left). In contrast, DANCE-MaP reveals a specific and near-complete network of direct PAIR interactions that clearly define the $\mathrm{ON}$ and OFF state secondary structures (Fig. 2B, 2C, right; in blue). Remarkably, DANCE identifies base pairing interactions that are invisible in the composite data (Fig. 2B, 2C; arrows). Minor PAIR signals further reveal dynamics hidden by per-nucleotide analysis, including the known dynamics (Reining et al., 2013) of the P2/P2alt region in the OFF state. Equally striking, DANCE-MaP detected through-space tertiary interactions (RINGs) clearly identifying the L2-L3 loop-loop interaction in the $\mathrm{ON}$ state (Fig. 2B, 2C, right; in red and orange). Our data are consistent with other studies (Warhaut et al., 2017) showing that this tertiary interaction forms 
even in the absence of adenine ligand. Additional RINGs observed at the 3' end of the ON state likely reflect further minor states. These data were reproducible across the comprehensive adenine titration (Fig. S3).

In sum, DANCE-MaP directly measures macrostate heterogeneity, base pairing, and tertiary interactions for each state in a complex ensemble, enabling complete structural analysis within a single, concise chemical probing experiment.

\section{Native 7SK RNA exists as a multi-state structural ensemble}

Motivated by the fundamental role of 7SK RNA in transcriptional regulation and prior evidence of 7SK dynamics (Krueger et al., 2010; Flynn et al., 2016; Brogie and Price, 2017), we sought to define the 7SK structural ensemble and its role in regulating transcription. We performed DMSMaP experiments on living human Jurkat cells and obtained high-coverage single-molecule DMS probing data for the 7SK RNA. Conventional averaged analysis (without deconvolution) yielded per-nucleotide reactivity profiles generally compatible with previously proposed SL1, SL3, and SL4 stem-loop structures (Wassarman and Steitz, 1991; Marz et al., 2009) (Fig. 3A, top). However, as observed previously (Wassarman and Steitz, 1991; Krueger et al., 2010; Brogie and Price, 2017; Wang et al., 2019), many nucleotides exhibit intermediate reactivities, consistent with significant, unresolved structural heterogeneity.

$\mathrm{ML}$ single-molecule analysis indicated that 7SK structural heterogeneity reproducibly resolves into three states: $\mathrm{A}, \mathrm{B}$, and $\mathrm{H}$ (populations of $40 \% \pm 3,47 \% \pm 2,13 \% \pm 2$, respectively). State populations and reactivity profiles $(R>0.96)$ were highly reproducible over 10 biological replicates performed years apart. The minority $H$ (heterogenous) state shares some features with $B$, but generally has high reactivity across the RNA. By comparison, the predominant $A$ and $B$ states show punctate regions of high and low nucleotide reactivity, consistent with these states representing well-defined structural states (Fig. 3A). Nucleotides throughout the SL1 region including $\mathrm{U} 28, \mathrm{U} 30, \mathrm{U} 66$, and $\mathrm{U} 68$ are unreactive in state $A$, but reactive in states $B$ and $\mathrm{H}$, corresponding precisely to nucleotides previously identified as changing conformation upon P-TEFb release (Krueger et al., 2010; Brogie and Price, 2017). Numerous additional differences occur throughout the 7SK RNA, indicative of a concerted global structural switch.

We repeated our experiments on protein-free RNA extracted from Jurkat cells that was heat denatured and refolded (referred to as cell-free). ML deconvolution revealed that cell-free 7SK 
also populates 3 states: $A$ and $B$, and a mixed $(M)$ state that shares features of both $A$ and $B$ (populations $43 \% \pm 8,29 \% \pm 6,28 \% \pm 1$; $\mathrm{R}>0.98$ between reactivity profiles; two consolidated replicates, see Methods). States $A$ and $B$ are the same as observed in-cell $(R=0.96$ and $R=$ 0.95 for states $A$ and $B$, respectively Fig. 3B). Only diffuse reactivity protections and enhancements are observed relative to the in-cell RNA, which implies that the $A$ and $B$ states are dynamically rather than stably bound by proteins in cells. The lack of an $\mathrm{H}$ state under cellfree conditions is consistent with $\mathrm{H}$ representing a state heterogeneously bound by proteins in cells. Conversely, the lack of a mixed state in cells suggests that bound cellular factors specifically favor the A and B states. Thus, the 7SK RNA sequence intrinsically codes for two energetically balanced states, specifying a large-scale structural switch that behaves similarly with or without bound proteins.

\section{Direct base pair mapping and structure modeling reveals 7SK architecture}

To fully resolve the secondary structure and potential tertiary structures of each state, we obtained high depth sequencing datasets (>3 million reads) that provide power sufficient to detect through-space PAIRs and RINGs across the $332 \mathrm{nt}$ long 7SK RNA. These data reveal numerous PAIR signals that directly report base-paired structural elements distinctive to each state (Fig. 3C), reproducible between in-cell and cell-free environments, and across consolidated replicates (Fig. S6). We used these PAIR data in combination with per-nucleotide reactivity profiles to build detailed secondary structure models for the $A$ and $B$ states (Fig. 3D). The resulting structural models reveal that $7 \mathrm{SK}$ folds into two globally different conformations, each of which is supported by distinctive per-nucleotide reactivities and state-specific, direct PAIR correlations. Both states also show alternative predicted pairing possibilities and PAIRs suggestive of residual heterogeneity (Fig. 3C), indicating that states $A$ and $B$ should be interpreted as class averages rather than pure states. Nevertheless, each state possesses key defining structural features, and these state-specific structures clearly support a link between 7SK conformational dynamics to P-TEFb binding and release.

\section{State $A$ is the P-TEFb binding-competent state with a dynamic SLO stem}

State A largely recapitulates classic models of 7SK structure, blending features predicted by early probing studies (Wassarman and Steitz, 1991) and more recent evolutionary analyses (Fig. S7) (Marz et al., 2009). The SL1 helix is the defining structural feature of state $A$, and is directly supported by PAIRs both in cells and for the cell-free extracted RNA (Fig. 3C). SL1 has been extensively validated as the recognition site for HEXIM1/2 and P-TEFb, based on in vitro 
binding assays (Lebars et al., 2010; Martinez-Zapien et al., 2016), analysis of P-TEFb-bound 7SK fractions from cells (Brogie and Price, 2017), and in-cell functional assays (Egloff et al., 2006; Fujinaga et al., 2014). The population of state A in cells, $40 \%$, is also consistent with the estimated fraction of 7SK bound by P-TEFb (Nguyen et al., 2001; Yang et al., 2001). Thus, we assign state $\mathrm{A}$ as the P-TEFb bound (sequestered) state.

Structure modeling indicates that this P-TEFb-sequestered state contains the long-range SL0 pairing interaction between the 5' and $3^{\prime}$ ends that "circularizes" the RNA (Marz et al., 2009). While we lack data for the $5^{\prime}$ strand of SLO due to overlap with the primer binding site, the $3^{\prime}$ strand of SLO is lowly-to-moderately reactive in-cells, consistent with formation of a dynamic, partially stable stem (Fig. 3, S8A). By contrast, the alternative extended form of SL1, which outcompetes SL0 in the cell-free RNA (SL1ext, see Fig. 3C), is reactive in cells, arguing against the "linear" structure. The increased stability of SLO in cells likely reflects favorable interactions with MePCE and LARP7, bound at the 5' and 3' ends of the RNA, respectively (Muniz et al., 2013; Eichhorn et al., 2018; Yang et al., 2019); indeed, SL0 pairing facilitates MePCE-LARP7 interactions in vitro (Brogie and Price, 2017). Given that SLO is strongly supported in state $B$ (Fig. 3A, S8A), our data thus indicate that the 7SK RNP primarily exists in a "circular" form in cells.

Additional structural features include the SL2, SL3, and SL4 stems, proposed in prior studies (Wassarman and Steitz, 1991; Marz et al., 2009; Brogie and Price, 2017). PAIR signals provide the first direct validation of the SL2 and SL3 stems (Fig. 3C, 3D). PAIR analysis further reveals a long-range SL3a interaction present for both in-cell and cell-free RNAs (Fig. 3C, 3D, left). Finally, we performed RING analysis to search for potential tertiary interactions that may stabilize the A state, analogous to those validated for the adenine riboswitch (Fig. 2B, 2C). Observed RINGs are relatively isolated (Fig. S6), and thus we see no compelling evidence for tertiary interactions in state $\mathrm{A}$.

\section{State $B$ is the P-TEFb released state with remodeled SL1 and central domains}

State B constitutes a novel structure without close literature precedent (Fig. 3C, S7). Most notably, SL1 is absent. Instead, this region folds into the previously postulated SL1alt stem (Krueger et al., 2010; Brogie and Price, 2017). Although overlap with the primer binding site precludes measurement of SL1alt-specific PAIRs, the disappearance of the SL1 PAIRs (as observed in state A) implies that this region adopts an alternative structure in state B. SL1alt, 
and not SL1, is also clearly supported by per-nucleotide DMS reactivities and by pairing probabilities (Fig. 3C). P-TEFb does not bind SL1alt (Czudnochowski et al., 2010; Fujinaga et al., 2014) and, indeed, P-TEFb binding converts SL1alt to SL1 in vitro (Brogie and Price, 2017). Conversely, release of P-TEFb induces conversion of SL1 to SL1alt (Krueger et al., 2010; Brogie and Price, 2017). The $47 \%$ population of state B in cells is also consistent with the fraction of 7SK that is in a P-TEFb-released state in cells (Nguyen et al., 2001; Yang et al., 2001). Thus, we conclude that state $B$ constitutes the P-TEFb released state.

SL1alt is coupled to formation of a major extension of SL2, which we term SL2ext, that has not been observed previously (Fig. 3C). Re-pairing to form SL2ext is directly supported by PAIRs in both in-cell and cell-free RNAs (Fig. 3C, blue arrows). Indeed, PAIR analysis was essential for resolving these interactions: SL2ext is not predicted when structure is modeled only on the basis of per-nucleotide reactivities (Fig. S8). Moderate DMS reactivities indicate that SL2ext is dynamic, and these dynamics are enhanced in cells, consistent with this region being bound by diverse proteins (Van Herreweghe et al., 2007; Ji et al., 2013; Flynn et al., 2016). Thus, while SL2ext is modeled as lowly probable in cells, the overall consistency between cell-free and incell PAIRs leads us to conclude SL2ext is present in state B in cells (Fig. 3D). The interdependence between SL1alt and SL2ext, visualized here, rationalizes prior observations that P-TEFb binding induces structural changes in the 7SK central region, located up to $200 \mathrm{nts}$ away from SL1 (Brogie and Price, 2017). As we discuss below, this structural reorganization overlaps the principal regions bound by P-TEFb release factors, consistent with allosteric coupling between SL1, SL2ext, and release factor binding sites.

Strikingly, RING analysis revealed a dense network of correlations for both cell-free and in-cell RNA (Fig. 3D, S6). Prior in vitro studies observed salt-dependent formation of an SL1altcontaining state, consistent with state $B$ potentially being stabilized by tertiary interactions (Brogie and Price, 2017). Some of these RINGs are likely indirect and reflect unresolved minor states. Nonetheless, the consistency and density of observed RINGs suggest that state B contains a compact central core stabilized by dynamic tertiary interactions.

\section{State $\boldsymbol{H}$ is a heterogenous $\boldsymbol{P}$-TEFb released state}

The (heterogenous) state $\mathrm{H}$ features well-defined SL0, SL1alt, and SL2 stems, but is otherwise highly reactive and contains no other stable structural elements (Fig. 3A, S8). The presence of SL1alt implies that, like $B$, state $H$ is a P-TEFb released state. However, the elevated reactivities 
within SL3 and the central domain indicate this state is highly heterogenous. Based on our analysis of simulated data (Fig. S2), we interpret this state to be a composite of diverse lowly populated protein-bound structures. One possibility is that $\mathrm{H}$ represents a transition state between $A$ and $B$ that is stabilized by helicases, which are known to bind to regions that are unstructured in $\mathrm{H}$ (see Discussion).

\section{The 7SK RNA ensemble comprises an allosteric switch}

In sum, our data reveal that 7SK folds into at least three structures that comprise P-TEFbcompetent and -released states. PAIR and RING measurements, measured individually for each state, provide pivotal and direct evidence of new structural elements (SL2ext) and a compact core in state B that are invisible to per-nucleotide analyses. This multi-state ensemble rationalizes a large compendium of biochemical and functional data on the 7SK RNP, and implies that 7SK contains an allosteric switch that couples HEXIM1/2-P-TEFb binding in SL1 to release factor binding sites in the central domain.

\section{Mutational analysis validates importance of SL2ext in 7SK structural switching}

We validated our DANCE-resolved models and defined the role of individual structural elements in 7SK switching using mutants to probe the A and B states. As an initial control, we performed DANCE-MaP experiments on in vitro transcripts of the native sequence RNA. The native RNA folds into a two-state ensemble (Fig. S9), consisting of the A and B states with populations $71 \pm$ 4 and $29 \pm 4 \%$, respectively ( $R>0.95$ between reactivity profiles; $N=3$ ). The two states are almost identical to the A and B states observed for the cell-free RNA (Fig. S9A), with the exception that SLO is further destabilized in State A. The lack of an M state in the in vitro transcribed RNA is also consistent with SLO destabilization. This destablization may reflect increased formal charge at the 5'-phosphate compared the endogenous transcript, which is 5'- $\gamma$ methylated (Jeronimo et al., 2007; Yang et al., 2019). These data from in vitro transcripts further validate that the $A$ and $B$ states are intrinsic features of the 7SK RNA sequence.

To validate the $B$ state structure, we introduced three mismatches in SL1 while leaving SL1alt pairing intact (mutant M1, Fig. 4A,B). DANCE-MaP experiments showed that M1 eliminates state $A$, with the RNA clustering into 2-3 B or $B$-like states (N=3; Fig. 4C, S9). Detection of multiple $B / B$-like states is consistent with the native $B$ state representing a composite of multiple similar structures, and with the M1 mutation modestly destabilizing SL1alt. Rescue of the M1 mutation by restoring base pairing complementarity in $S L 1(M 1+M 2)$ recovers the native $A: B$ 
equilibrium (78 \pm 2 and $22 \pm 2 \%$ populations, respectively; N=3; Fig. 4, S9B).

We next investigated the role of SL0 in 7SK switching. Our in-cell data support SL0 formation in both states, although SLO is clearly more stable in state B. Others have proposed that SL0 dynamics drive SL1:SL1alt switching (Brogie and Price, 2017). Ablation of SL0 via the M3 mutation (Fig. 4A) had minimal impact on the 7SK ensemble: M3 populates an $80 \pm 3,20 \pm 3 \%$ equilibrium consisting of 1-2 A/A-like states and a heterogenous B-like state that replaces SLO with other interactions ( $N=3$, Fig. S9B). Mutation of only three of seven base pairs in SL0 gave similar results (not shown). Thus, SLO does not drive the A:B equilibrium.

Finally, we examined the role of SL2ext in A:B switching. PAIR interactions directly support formation of the SL2ext stem for both cell-free and in-cell RNAs (Fig. 3C,D). We thus designed a mutant, M4, to disrupt the PAIR-supported 3-helix junction at the base of SL2ext in state B, but not perturb SL1 pairing in state A (Fig. 4A). Strikingly, this three-nucleotide mutation fully shifts the ensemble to A/A-like states (N=2; Fig. 4C, S9B). Prior studies have also observed that mutations in the SL2ext region induce global remodeling of 7SK structure (Brogie and Price, 2017; Luo et al., 2021), although the mechanistic basis was not resolved. Thus, even though SL2ext shows intermediate stability, this region is critical to 7SK A:B switching.

In sum, the 7SK ensemble can be rationally perturbed via structure-informed mutations, validating the DANCE-resolved A and B states. Moreover, a concise mutation to SL2ext (M4) is sufficient to drive SL1/SL1alt switching, establishing that the central core is an energetically accessible platform for modulating 7SK structure and activity.

\section{The 7SK structural equilibrium is regulated by cell type and transcriptional demands}

The 7SK-P-TEFb axis functions as a global regulator of transcription, and we hypothesized that the 7SK ensemble may be sensitive to cellular gene expression and growth needs. Jurkat cells, like many other tumor cells, exhibit altered P-TEFb regulation and aberrantly upregulated transcription compared to normal cells (Lin et al., 2003; Tyagi et al., 2010). We thus examined whether the 7SK ensemble differs in normal, non-transformed human RPE-1 cells (Bodnar et al., 1998). DANCE-MaP experiments performed on living RPE-1 cells revealed that 7SK adopts precisely the same three structures as observed for Jurkat cells (Fig. 5A) but with significantly different populations: states $A, B$, and $H$ have populations of $47 \pm 2,34 \pm 2$, and $19 \% \pm 2$, respectively, in RPE-1 cells compared to $40 \pm 3,47 \pm 2,13 \% \pm 1$ in Jurkat cells (N=7; Fig. 5B). 
This shift represents a $\sim 30 \%$ relative change in the $7 \mathrm{SK}$ equilibrium towards $\mathrm{A}$ and $\mathrm{H}$ and away from $B$, and is consistent with P-TEFb being more sequestered in RPE-1 cells (or aberrantly released in Jurkat tumor cells). We also probed 7SK in quiescent RPE-1 cells, where transcriptional load is expected to be further decreased. Indeed, the population of state A further increases to $54 \% \pm 1$ in contact-inhibited RPE- 1 cells (15\% relative increase compared to proliferating RPE-1 cells; N=3; Fig. 5B). In absolute molecular terms, each of these changes in 7SK equilibrium correspond to $\sim 10,0007$ SK snRNPs per cell shifting to a P-TEFb binding conformation (Gurney and Eliceiri, 1980; Wassarman and Steitz, 1991).

7SK and P-TEFb are also dynamically regulated in response to transcriptional stress (Peterlin et al., 2012; Quaresma et al., 2016). Flavopiridol is a pan-CDK inhibitor that suppresses transcription by inhibiting CDK9 (Chao et al., 2000). To compensate for reduced CDK9 activity, flavopiridol induces cells to release P-TEFb from 7SK, which conventional probing experiments have indicated induces structural changes in the 7SK RNA (Biglione et al., 2007; Krueger et al., 2010). We directly visualized these structural changes by performing DANCE-MaP experiments in Jurkat and RPE-1 cells treated with either vehicle (DMSO, 0.01\%) or $1 \mu \mathrm{M}$ (saturating) flavopiridol (Biglione et al., 2007). In both cell types, flavopiridol treatment dramatically remodels the 7SK structural ensemble. In Jurkat cells, state $A$ is completely converted to B/B-like states, consistent with total P-TEFb release (Fig. 5C). By contrast, RPE-1 cells exhibit significant, but partial, depopulation of state $\mathrm{A}(48 \%$ to $27 \%$ ) and conversion to state $\mathrm{H}$ (a non-P-TEFb-binding state) (Fig. 5C). This switch supports the physiological relevance of state $\mathrm{H}$ and indicates that the 7SK ensemble and P-TEFb release are governed by multiple cell-type-dependent pathways. The attenuated 7SK response to flavopiridol is consistent with the greater tolerance of RPE-1 cells to transcription inhibition as compared to transcription-addicted (Kwiatkowski et al., 2014) Jurkat cells. Collectively, these data establish that the 7SK conformational equilibrium is tunable, is cell type-specific, and remodels dynamically, coincident with P-TEFb release.

\section{ASO stabilization of state B induces transcription in cells}

Our data emphasize that the 7SK structural switch is centrally linked to P-TEFb release and transcription regulation, motivating us to explore the potential of targeting the 7SK ensemble as a strategy for controlling transcription. After screening multiple candidates, we identified an antisense oligonucleotide, ASO-B, that disrupts state $A$ without impacting the major helices unique to state $B$ (Fig. 6A). DANCE-MaP experiments on cell-free RNA confirmed that ASO-B shifted the 7SK structural ensemble to exclusively B/B-like states, whereas a control ASO 
containing five central mismatches (MM-B) had no significant impact on the 7SK ensemble (Fig. 6B, C). Thus, ASO-B constitutes a molecular tool for modulating the 7SK structural ensemble.

We then tested the ability of ASO-B to modulate transcription in cells. We first confirmed ASO delivery and engagement in HEK293 cells using a gapmer oligonucleotide (GAP-B) targeting the same region of $7 \mathrm{SK}$ but designed to induce $\mathrm{RNase} \mathrm{H}$ degradation. Treatment with GAP-B reduced 7 SK levels by 31 and $36 \%$ at $100 \mathrm{nM}$ and $200 \mathrm{nM}$ concentrations, respectively (Fig. 6D). To assess the impact of 7SK depletion on transcription, we measured the cellular levels of HEXIM1 RNA, which is a sensitive reporter of P-TEFb-mediated transcription activity (He et al., 2006; Liu et al., 2014). As expected, GAP-B-mediated depletion of 7SK induced 1.7- and 2.6fold induction of HEXIM1 mRNA expression, respectively (Fig. 6D). These data confirm in-cell engagement with 7 SK by the ASO-B sequence. We then tested the ability of ASO-B to induce P-TEFb release via stabilization of 7 SK state B. Treatment with 100 nM ASO-B but not MM-B yielded a 1.7-fold increase in HEXIM1, without impacting 7SK expression (Fig. 6E), directly validating that 7SK switching induces $\mathrm{P}-\mathrm{TEFb}$ release. We further examined whether ASOmediated structure-switching could complement transcriptional activation by the small molecule JQ1. JQ1 is a bromodomain and extra-terminal (BET) inhibitor that induces P-TEFb release via a 7SK-independent mechanism (Chaidos et al., 2014; Fujinaga et al., 2015). Indeed, co-addition of 100 nM JQ1 increased HEXIM1 expression 3.3-fold, comparable to the upregulation observed upon treatment with $250 \mathrm{nM}$ JQ1 alone (Fig. 6E). Together, these experiments further establish a causal relationship between 7SK structural switching and P-TEFb release and provide proof of principle for targeting the 7SK structural switch.

\section{Discussion}

\section{DANCE-MaP enables complete analysis of RNA structural ensembles}

Most RNAs have the potential to fold into multiple structures, which creates numerous opportunities to regulate RNA biology. However, authoritatively defining RNA structural ensembles in cells and their responses to cellular stimuli has remained a critical unresolved challenge. We introduce DANCE-MaP, a single-molecule chemical probing technology that simultaneously measures per-nucleotide reactivities, through-space base pairs (PAIRs), and tertiary interactions (RINGs) for multiple co-existing RNA structural states in cells. DANCE-MaP further measures populations with thermodynamic precision, enabling measurement of ligand binding affinity and of subtle but impactful structural differences between cell types. Collectively, 
these integrated measurements enable definitive and comprehensive characterization of RNA structural ensembles.

Our study emphasizes the intrinsic complexity of RNA structural ensembles. For the adenine riboswitch, the ON and OFF states are defined by the presence of the aptamer domain and the SD-sequestering helix, respectively, but exhibit substantial heterogeneity elsewhere in the molecule. These data are consistent with and clarify observations from prior biophysical and chemical probing studies (Reining et al., 2013; Warhaut et al., 2017; Tian et al., 2018; Tomezsko et al., 2020). For 7SK, the A, B, and $\mathrm{H}$ states are each distinguished by major structural landmarks, but ultimately represent class averages rather than singular conformations. Within this context of underlying heterogeneity, the ability of DANCE-MaP to measure base pairing interactions directly and to estimate pairing probabilities within each state is a crucial advance, enabling us to resolve otherwise invisible dynamic structures in cells and to model global RNA architectures with confidence. Direct PAIR measurements were essential for resolving the SL2ext structure unique to state B of 7SK. RINGs measured in the compact core of state B further emphasize this state has a distinct higher-order structure. Integrated DANCE$\mathrm{MaP}$ analysis represents a powerful advance over single-purpose deconvolution or duplex detection strategies and will broadly enable studies of RNA dynamic complexity in cells.

DANCE-MaP does have several limitations. While our data and other studies (Homan et al., 2014; Mustoe et al., 2019; Tomezsko et al., 2020; Luo et al., 2021; Morandi et al., 2021) support that multiple-hit DMS modification experiments accurately report native RNA structure, accumulated chemical damage may alter behavior of some RNAs. DANCE-MaP can only resolve structural changes that involve $>20$ nucleotides with populations of at least $\sim 5 \%$, and currently has a length limit of $\sim 500$ nucleotides across a single strand of RNA. DANCE-MaP has a time-resolution of $\sim 5$ minutes using DMS, but $\sim 10$ second resolution is possible with newer reagents (Ehrhardt and Weeks, 2020). DANCE-MaP further requires that each read originate from a unique RNA molecule, and thus will be more challenging to implement for low abundance RNAs.

Ultimately, DANCE-MaP provides many of the same measurements previously accessible only using state-of-art the NMR experiments, which have provided the primary ground-truth references for RNA ensembles (Liu et al., 2021). The tertiary RINGs measured in the ON state of the adenine riboswitch are of sufficient quality to guide accurate three-dimensional structure 
modeling (Homan et al., 2014; Li et al., 2020). The RINGs measured in 7SK State B are more challenging to interpret due to the residual dynamics of this state, but such dynamics would similarly challenge established biophysical techniques. Uniquely, DANCE-MaP is readily performed in cells on endogenous RNAs, and requires modest experimental effort. DANCEMaP thus paves the way for a new generation of biophysical studies in living systems.

\section{Allostery couples 7SK HEXIM1-P-TEFb aptamer domain to release factor binding sites} Regulated release of P-TEFb from the 7SK snRNP to phosphorylate Pol II is a critical control point in transcription (Peterlin et al., 2012). We show that the 7SK RNA intrinsically encodes a large-scale structural switch that modulates its P-TEFb binding ability (Fig. 7). We further find that the 7SK structural equilibrium is actively controlled by the cell in response to transcriptional demands. Quiescence favors a P-TEFb-sequestered state compared to proliferating cells, and transcriptional stress (CDK9 inhibition) favors a P-TEFb released state (Fig. 5). These population changes correspond to the release or sequestration of tens of thousands of P-TEFb molecules per cell. The amount of free P-TEFb is roughly equivalent to the number of engaged Pol II molecules in a cell (Gurney and Eliceiri, 1980; Kimura et al., 1999; Nguyen et al., 2001; Yang et al., 2001), suggesting that these 7SK/P-TEFb changes play a major role in reshaping global transcription. We also uncover significant changes in the 7SK equilibrium in cancer versus normal cells, providing one of the first examples of cell-type dependence in RNA structure. Collectively, these data support that the 7SK structural switch functions as a key axis of transcriptional regulation.

Numerous proteins have been implicated as "release factors" that stimulate P-TEFb release from 7SK, a group that includes both helicases and general RNA binding proteins (Van Herreweghe et al., 2007; Ji et al., 2013; Calo et al., 2015; Mück et al., 2016; Bugai et al., 2019; Sithole et al., 2020). Puzzlingly, , these proteins bind 7SK hundreds of nucleotides away from the SL1 hairpin, which comprises the binding site for HEXIM1/2 and P-TEFb (HEXIM/P-TEFb aptamer domain; Fig. 7) (Egloff et al., 2006; Krueger et al., 2008; Martinez-Zapien et al., 2016; Roder et al., 2019). Several studies have observed that HEXIM/P-TEFb release coincides with conformational changes in the SL1 aptamer (Krueger et al., 2010; Brogie and Price, 2017). Binding of hnRNPs has also been observed to induce conformational changes in the 7SK central domain (Luo et al., 2021). However, the relationship between these conformational changes and their role in P-TEFb release remained unclear. Our data resolve this puzzle and support a model whereby release factors catalyze 7SK structural switching and thereby 
allosterically effect P-TEFb release (Fig. 7). Significantly, release factor binding sites directly overlap or are immediately adjacent to the SL2ext and compact core structures in state B, ideally positioning them to effect structure switching. Given that the unstructured regions of state $\mathrm{H}$ also overlap these release factor binding sites, state $\mathrm{H}$ may represent an intermediate along the A to B pathway.

This allosteric model of P-TEFb release unifies multiple prior observations, as allostery: ( $i$ ) enables 7SK to maintain a specialized "release domain" that can integrate cellular signals unencumbered by bound P-TEFb; and (ii) prevents P-TEFb reassociation once release is triggered. The importance of role (ii) is supported by the observation that HIV-1 Tat, despite directly abstracting P-TEFb without binding the 7SK release domain, also induces conformational changes in 7SK (Krueger et al., 2010), which can now be interpreted as switching from state $A$ to $B$. Less is known regarding how $7 S K$ resequesters $P$-TEFb. We posit that helicases stimulate disassociation of hnRNPs and remodel 7SK to state A (the SL1containing form), enabling HEXIM/P-TEFb to rebind. Given that 7SK may be involved in transcription termination (Castelo-Branco et al., 2013), this process may be linked to Pol II recycling. In this model, distinct sets of helicases and other RNA binding proteins catalyze 7SK switching between structural states to either sequester or release P-TEFb.

The allosteric switching model also rationalizes the extreme sequence conservation of the first 100 7SK nucleotides across vertebrates and invertebrates (Yazbeck et al., 2018), which must preserve both HEXIM/P-TEFb binding and the dual constraints of forming the distinct SL1 and SL1alt structures. By comparison, the compact core region is highly conserved among Tetrapoda, supporting its functional importance, but diverges outside of Tetrapoda, suggesting that there are multiple ways to create a P-TEFb-regulating allosteric switch. This pattern of a highly conserved P-TEFb aptamer and variable core is also observed in classic riboswitches, where conserved aptamer domains are often integrated with diverse expression domain architectures (Roth and Breaker, 2009).

\section{SK structural switch links P-TEFb release to pro-transcription functions}

7SK is canonically considered a transcriptional repressor due to its P-TEFb sequestering function. However, the 7SK snRNP also has pro-transcription functions, including blocking convergent transcription via association with the BAF complex (Flynn et al., 2016) and facilitating spliceosome production (Egloff et al., 2017; Ji et al., 2021). These pro-transcription 
functions are specific to 7SK snRNPs lacking P-TEFb. Notably, prior chemical probing data obtained for BAF-associated 7SK (Flynn et al., 2016) can now be interpreted as corresponding to state $\mathrm{B}$ or $\mathrm{H}$. Together, these data support an overarching model in which the 7SK structural switch integrates P-TEFb release with conversion of $7 \mathrm{SK}$ into a pro-transcription snRNP that scaffolds assembly of elongation-supporting factors (Fig. 7, right). Significantly, in this model, 7SK switching would enable spatial and temporal coupling between Pol II pause release and BAF-mediated inhibition of convergent transcription (Flynn et al., 2016). Our dual-function model also rationalizes observations that 7SK is inessential for basal P-TEFb regulation (Studniarek et al., 2021), but that 7SK depletion perturbs global chromatin structure (Prasanth et al., 2010) and compromises stress-induced transcriptional reprogramming (Studniarek et al., 2021).

Overall, our model (Fig. 7) emphasizes how structural switching enables the 7SK snRNP to integrate diverse signals to cooperatively inactivate or activate transcription in response to cellular demand. RNAs are unique among biomolecules in their ability to encode large but precise changes in structure (Breaker, 2012; Dethoff et al., 2012). Coupled with the potential of RNAs to form aptamer domains, this makes RNAs optimally suited to serve as molecular integrators. We speculate that similar switching mechanisms broadly underlie non-coding RNA regulatory function.

\section{SK switch constitutes a novel therapeutic target for modulating transcription}

Our study shows that the 7SK structural equilibrium is regulated in response to changing transcription needs. We further show using proof-of-principle ASO studies that switching the 7SK state induces transcription of P-TEFb-sensitive targets. Developing small molecules or improved ASOs that stabilize state $B$, thereby releasing P-TEFb and activating transcription, represents a component of a promising strategy to eradicate persistent HIV infection by inducing the expression of latent provirus (Richman et al., 2009; Cary et al., 2016). Conversely, $\mathrm{P}-\mathrm{TEFb}$ is commonly dysregulated in cancer and there is intense interest in developing pharmacological inhibitors of P-TEFb as a cancer therapeutic (Yang et al., 2020). Disruption of the 7SK/P-TEFb regulatory axis has been linked to tumorigenesis and cancer progression (Cheng et al., 2012; Ji et al., 2014; Tan et al., 2016), consistent with a model in which dysregulation of the 7SK structural equilibrium supports elevated transcription in cancer cells. Designing small molecules or ASOs that reduce the cellular availability of P-TEFb by selectively stabilizing 7 SK state A represents a compelling therapeutic hypothesis for targeting transcription in cancer. 


\section{Acknowledgements}

We thank Khoa Dhoa (Baylor College of Medicine, BCM) for exploratory analyses, and Calla Olson (BCM), Kristen Karlin (BCM), and David Price (University of lowa) for helpful discussions. Research reported in this publication was supported by Qura Therapeutics (D.M.M.), by the NIH (R35 GM122532 to K.M.W), the Cancer Prevention \& Research Institute of Texas (RR190054 to A.M.M.), and BCM seed funds (to A.M.M.). The content of this study is solely the responsibility of the authors and does not necessarily represent the official views of Qura Therapeutics.

\section{Disclaimer}

A.M.M. is a consultant to, and K.M.W. is an advisor to and holds equity in Ribometrix. 


\section{References}

Barboric, M., Lenasi, T., Chen, H., Johansen, E.B., Guo, S., and Peterlin, B.M. (2009). 7SK snRNP/P-TEFb couples transcription elongation with alternative splicing and is essential for vertebrate development. Proc. Natl. Acad. Sci. U. S. A. 106, 7798-7803.

Biglione, S., Byers, S.A., Price, J.P., Nguyen, V.T., Bensaude, O., Price, D.H., and Maury, W. (2007). Inhibition of HIV-1 replication by P-TEFb inhibitors DRB, seliciclib and flavopiridol correlates with release of free P-TEFb from the large, inactive form of the complex. Retrovirology 4, 1-12.

Bishop, C. (2006). Pattern Recognition and Machine Learning (Springer).

Bodnar, A.G., Ouellette, M., Frolkis, M., Holt, S.E., Morin, G.B., Harley, C.B., Shay, J.W., Lichsteiner, S., Wright, E., Bodnar, A.G., et al. (1998). Extension of Life-Span by Introduction of Telomerase into Normal Human Cells. Science (80-. ). 279, 349-352.

Breaker, R.R. (2012). Riboswitches and the RNA world. Cold Spring Harb. Perspect. Biol. 4. Brogie, J.E., and Price, D.H. (2017). Reconstitution of a functional 7SK snRNP. Nucleic Acids Res. 1-17.

Bugai, A., Quaresma, A.J.C., Friedel, C.C., Lenasi, T., Düster, R., Sibley, C.R., Fujinaga, K., Kukanja, P., Hennig, T., Blasius, M., et al. (2019). P-TEFb Activation by RBM7 Shapes a Prosurvival Transcriptional Response to Genotoxic Stress. Mol. Cell 74, 254-267.e10.

Busan, S., and Weeks, K.M. (2018). Accurate detection of chemical modifications in RNA by mutational profiling (MaP) with ShapeMapper 2. Rna 24, 143-148.

Calo, E., Flynn, R.A., Martin, L., Spitale, R.C., Chang, H.Y., and Wysocka, J. (2015). RNA helicase DDX21 coordinates transcription and ribosomal RNA processing. Nature 518, 249253.

Cary, D.C., Fujinaga, K., and Peterlin, B.M. (2016). Molecular mechanisms of HIV latency. J. Clin. Invest. 126, 448-454.

Castelo-Branco, G., Amaral, P.P., Engström, P.G., Robson, S.C., Marques, S.C., Bertone, P., and Kouzarides, T. (2013). The non-coding snRNA 7SK controls transcriptional termination, poising, and bidirectionality in embryonic stem cells. Genome Biol. 14.

Cech, T.R., and Steitz, J.A. (2014). The noncoding RNA revolution - Trashing old rules to forge new ones. Cell 157, 77-94.

Chaidos, A., Caputo, V., Gouvedenou, K., Liu, B., Marigo, I., Chaudhry, M.S., Rotolo, A., Tough, D.F., Smithers, N.N., Bassil, A.K., et al. (2014). Potentantimyeloma activity of the novel bromodomain inhibitors I-BET151 and I-BET762. Blood 123, 697-705.

Chao, S.H., Fujinaga, K., Marion, J.E., Taube, R., Sausville, E.A., Senderowicz, A.M., Peterlin, B.M., and Price, D.H. (2000). Flavopiridol inhibits P-TEFb and blocks HIV-1 replication. J. Biol. Chem. 275, 28345-28348.

Cheng, C.Y., Kladwang, W., Yesselman, J.D., and Das, R. (2017). RNA structure inference through chemical mapping after accidental or intentional mutations. Proc. Natl. Acad. Sci. U. S. A. 114, 9876-9881.

Cheng, Y., Jin, Z., Agarwal, R., Ma, K., Yang, J., Ibrahim, S., Olaru, A. V., David, S., Ashktorab, H., Smoot, D.T., et al. (2012). LARP7 is a potential tumor suppressor gene in gastric cancer. Lab. Investig. 92, 1013-1019. 
Czudnochowski, N., Vollmuth, F., Baumann, S., Vogel-Bachmayr, K., and Geyer, M. (2010). Specificity of Hexim1 and Hexim2 Complex Formation with Cyclin T1/T2, Importin a and 7SK snRNA. J. Mol. Biol. 395, 28-41.

Dethoff, E.A., Chugh, J., Mustoe, A.M., and Al-Hashimi, H.M. (2012). Functional complexity and regulation through RNA dynamics. Nature 482, 322-330.

Dethoff, E.A., Boerneke, M.A., Gokhale, N.S., Muhire, B.M., Martin, D.P., Sacco, M.T., McFadden, M.J., Weinstein, J.B., Messer, W.B., Horner, S.M., et al. (2018). Pervasive tertiary structure in the dengue virus RNA genome. Proc. Natl. Acad. Sci. U. S. A. 115, 11513-11518.

Egloff, S., Van Herreweghe, E., and Kiss, T. (2006). Regulation of Polymerase II Transcription by 7SK snRNA: Two Distinct RNA Elements Direct P-TEFb and HEXIM1 Binding. Mol. Cell. Biol. 26, 630-642.

Egloff, S., Vitali, P., Tellier, M., Raffel, R., Murphy, S., and Kiss, T. (2017). The 7SK snRNP associates with the little elongation complex to promote snRNA gene expression. EMBO J. 36, 934-948.

Ehrhardt, J.E., and Weeks, K.M. (2020). Time-Resolved, Single-Molecule, Correlated Chemical Probing of RNA. J. Am. Chem. Soc. 142, 18735-18740.

Eichhorn, C.D., Yang, Y., Repeta, L., and Feigon, J. (2018). Structural basis for recognition of human 7SK long noncoding RNA by the La-related protein Larp7 [Biophysics and Computational Biology]. Pnas 1-10.

Eilebrecht, S., Brysbaert, G., Wegert, T., Urlaub, H., Benecke, B.J., and Benecke, A. (2011). 7SK small nuclear RNA directly affects HMGA1 function in transcription regulation. Nucleic Acids Res. 39, 2057-2072.

Flynn, R.A., Do, B.T., Rubin, A.J., Calo, E., Lee, B., Kuchelmeister, H., Rale, M., Chu, C., Kool, E.T., Wysocka, J., et al. (2016). 7SK-BAF axis controls pervasive transcription at enhancers. Nat. Struct. Mol. Biol. 23, 231-238.

Fu, Y., Deiorio-Haggar, K., Anthony, J., and Meyer, M.M. (2013). Most RNAs regulating ribosomal protein biosynthesis in Escherichia coli are narrowly distributed to Gammaproteobacteria. Nucleic Acids Res. 41, 3491-3503.

Fujinaga, K., Luo, Z., and Peterlin, B.M. (2014). Genetic analysis of the structure and function of 7SK small nuclear ribonucleoprotein (snRNP) in cells. J. Biol. Chem. 289, 21181-21190.

Fujinaga, K., Luo, Z., Schaufele, F., and Matija Peterlin, B. (2015). Visualization of positive transcription elongation factor b (P-TEFb) activation in living cells. J. Biol. Chem. 290, 18291836.

Gurney, T., and Eliceiri, G.L. (1980). Intracellular distribution of low molecular weight RNA species in hela cells. J. Cell Biol. 87, 398-403.

Hajdin, C.E., Bellaousov, S., Huggins, W., Leonard, C.W., Mathews, D.H., and Weeks, K.M. (2013). Accurate SHAPE-directed RNA secondary structure modeling, including pseudoknots. Proc. Natl. Acad. Sci. U. S. A. 110, 5498-5503.

He, N., Pezda, A.C., and Zhou, Q. (2006). Modulation of a P-TEFb Functional Equilibrium for the Global Control of Cell Growth and Differentiation. Mol. Cell. Biol. 26, 7068-7076.

Van Herreweghe, E., Egloff, S., Goiffon, I., Jády, B.E., Froment, C., Monsarrat, B., and Kiss, T. (2007). Dynamic remodelling of human 7SK snRNP controls the nuclear level of active P-TEFb. EMBO J. 26, 3570-3580. 
Homan, P.J., Favorov, O. V., Lavender, C.A., Kursun, O., Ge, X., Busan, S., Dokholyan, N. V., and Weeks, K.M. (2014). Single-molecule correlated chemical probing of RNA. Proc. Natl. Acad. Sci. U. S. A. 111, 13858-13863.

Jeronimo, C., Forget, D., Bouchard, A., Li, Q., Chua, G., Poitras, C., Thérien, C., Bergeron, D., Bourassa, S., Greenblatt, J., et al. (2007). Systematic Analysis of the Protein Interaction Network for the Human Transcription Machinery Reveals the Identity of the 7SK Capping Enzyme. Mol. Cell 27, 262-274.

Ji, C., Bader, J., Ramanathan, P., Hennlein, L., Meissner, F., Jablonka, S., Mann, M., Fischer, U., Sendtner, M., and Briese, M. (2021). Interaction of 7SK with the Smn complex modulates snRNP production. Nat. Commun. 12.

Ji, X., Yu, Z., Pandit, S., Jie, H., Li, H., Lin, C.Y., Xiao, R., Burge, C.B., and Fu, X.-D. (2013). SR Proteins Collaborate with 7SK and Promoter-Associated Nascent RNA to Release Paused Polymerase. Cell 153, 855-868.

Ji, X., Lu, H., Zhou, Q., and Luo, K. (2014). LARP7 suppresses P-TEFb activity to inhibit breast cancer progression and metastasis. Elife 3, e02907.

Kalvari, I., Nawrocki, E.P., Ontiveros-Palacios, N., Argasinska, J., Lamkiewicz, K., Marz, M., Griffiths-Jones, S., Toffano-Nioche, C., Gautheret, D., Weinberg, Z., et al. (2021). Rfam 14: Expanded coverage of metagenomic, viral and microRNA families. Nucleic Acids Res. 49, D192-D200.

Kimura, H., Tao, Y., Roeder, R.G., and Cook, P.R. (1999). Quantitation of RNA Polymerase II and Its Transcription Factors in an HeLa Cell: Little Soluble Holoenzyme but Significant Amounts of Polymerases Attached to the Nuclear Substructure. Mol. Cell. Biol. 19, 5383-5392.

Krokhotin, A., Mustoe, A.M., Weeks, K.M., and Dokholyan, N. V (2016). Direct identification of base-paired RNA nucleotides by correlated chemical probing. RNA.

Krueger, B.J., Jeronimo, C., Roy, B.B., Bouchard, A., Barrandon, C., Byers, S.A., Searcey, C.E., Cooper, J.J., Bensaude, O., Cohen, É.A., et al. (2008). LARP7 is a stable component of the 7SK snRNP while P-TEFb, HEXIM1 and hnRNP A1 are reversibly associated. Nucleic Acids Res. 36, 2219-2229.

Krueger, B.J., Varzavand, K., Cooper, J.J., and Price, D.H. (2010). The mechanism of release of P-TEFb and HEXIM1 from the 7SK snRNP by viral and cellular activators includes a conformational change in 7SK. PLoS One 5.

Kwiatkowski, N., Zhang, T., Rahl, P.B., Abraham, B.J., Reddy, J., Ficarro, S.B., Dastur, A., Amzallag, A., Ramaswamy, S., Tesar, B., et al. (2014). Targeting transcription regulation in cancer with a covalent CDK7 inhibitor. Nature 511, 616-620.

Lebars, I., Durand, A., Coutant, J., Kieffer, B., Fries, L., Cedex, I., Sa, B.B., Industrie, D., Martinez-Zapien, D., Durand, A., et al. (2010). HEXIM1 targets a repeated GAUC motif in the riboregulator of transcription 7 SK and promotes base pair rearrangements. Nucleic Acids Res. 38, 7749-7763.

Li, B., Cao, Y., Westhof, E., and Miao, Z. (2020). Advances in RNA 3D Structure Modeling Using Experimental Data. Front. Genet. 11, 1-19.

Lin, Z., Fillmore, G.C., Um, T.H., Elenitoba-Johnson, K.S.J., and Lim, M.S. (2003). Comparative microarray analysis of gene expression during activation of human peripheral blood $\mathrm{T}$ cells and leukemic Jurkat T cells. Lab. Investig. 83, 765-776.

Liu, B., Shi, H., and Al-Hashimi, H.M. (2021). Developments in solution-state NMR yield broader 
and deeper views of the dynamic ensembles of nucleic acids. Curr. Opin. Struct. Biol. 70, 1625.

Liu, P., Xiang, Y., Fujinaga, K., Bartholomeeusen, K., Nilson, K.A., Price, D.H., and Peterlin, B.M. (2014). Release of positive transcription elongation factor $b$ (P-TEFb) from 7SK small nuclear ribonucleoprotein (snRNP) activates hexamethylene bisacetamide-inducible protein (HEXIM1) transcription. J. Biol. Chem. 289, 9918-9925.

Luo, L., Chiu, L., Sugarman, A., Gupta, P., Rouskin, S., and Tolbert, B.S. (2021). HnRNP A1/A2 Proteins Assemble onto 7SK snRNA via Context Dependent Interactions. J. Mol. Biol. 166885.

Martinez-Zapien, D., Legrand, P., McEwen, A.G., Proux, F., Cragnolini, T., Pasquali, S., and Dock-Bregeon, A.C. (2016). The crystal structure of the 5' functional domain of the transcription riboregulator 7SK. Nucleic Acids Res. 45, 3568-3579.

Marz, M., Donath, A., Verstraete, N., Nguyen, V.T., Stadler, P.F., and Bensaude, O. (2009). Evolution of 7SK RNA and its protein partners in metazoa. Mol. Biol. Evol. 26, 2821-2830.

Matson, J.P., House, A.M., Grant, G.D., Wu, H., Perez, J., and Cook, J.G. (2019). Intrinsic checkpoint deficiency during cell cycle re-entry from quiescence. J. Cell Biol. 218, 2169-2184.

McLachlan, G., and Peel, D. (2000). Finite Mixture Models (New York: Wiley).

Michael W. Pfaffl (2001). A new mathematical model for relative quantification in real-time RTPCR. Mon. Not. R. Astron. Soc. 29, 2002-2007.

Morandi, E., Manfredonia, I., Simon, L.M., Anselmi, F., van Hemert, M.J., Oliviero, S., and Incarnato, D. (2021). Genome-scale deconvolution of RNA structure ensembles. Nat. Methods 18.

Mück, F., Bracharz, S., and Marschalek, R. (2016). DDX6 transfers P-TEFb kinase to the AF4/AF4N (AFF1) super elongation complex. Am. J. Blood Res. 6, 28-45.

Muniz, L., Egloff, S., and Kiss, T. (2013). RNA elements directing in vivo assembly of the 7SK/MePCE/Larp7 transcriptional regulatory snRNP. Nucleic Acids Res. 41, 4686-4698.

Mustoe, A.M., Lama, N.N., Irving, P.S., Olson, S.W., and Weeks, K.M. (2019). RNA basepairing complexity in living cells visualized by correlated chemical probing. Proc. Natl. Acad. Sci. U. S. A. 116, 24574-24582.

Nguyen, V.T., Kiss, T., Michels, A.A., and Bensaude, O. (2001). 7SKsmall nuclear RNA binds to and inhibits the activity of CDK9/cyclin T complexes. Nature 414, 322-325.

Olson, C.M., Jiang, B., Erb, M.A., Liang, Y., Doctor, Z.M., Zhang, Z., Zhang, T., Kwiatkowski, N., Boukhali, M., Green, J.L., et al. (2018). Pharmacological perturbation of CDK9 using selective CDK9 inhibition or degradation. Nat. Chem. Biol. 14, 163-170.

Peterlin, B.M., and Price, D.H. (2006). Controlling the Elongation Phase of Transcription with PTEFb. Mol. Cell 23, 297-305.

Peterlin, B.M., Bragie, J., Price, D.D.H., Brogie, J.E., and Price, D.D.H. (2012). 7SK snRNA: A noncoding RNA that plays a major role in regulating eukaryotic transcription. Wiley Interdiscip. Rev. RNA 3, 92-103.

Prasanth, K. V., Camiolo, M., Chan, G., Tripathi, V., Denis, L., Nakamura, T., Hübner, M.R., and Spector, D.L. (2010). Nuclear organization and dynamics of 7SK RNA in regulating gene expression. Mol. Biol. Cell 21, 4184-4196.

Quaresma, A.J.C., Bugai, A., and Barboric, M. (2016). Cracking the control of RNA polymerase II elongation by 7SK snRNP and P-TEFb. Nucleic Acids Res. 44, 7527-7539. 
Ray, P.S., Jia, J., Yao, P., Majumder, M., Hatzoglou, M., and Fox, P.L. (2009). A stressresponsive RNA switch regulates VEGFA expression. Nature 457, 915-919.

Reining, A., Nozinovic, S., Schlepckow, K., Buhr, F., Fürtig, B., and Schwalbe, H. (2013). Threestate mechanism couples ligand and temperature sensing in riboswitches. Nature 499, 355359.

Reuter, J.S., and Mathews, D.H. (2010). RNAstructure: Web servers for RNA secondary structure prediction and analysis. BMC Bioinformatics.

Richman, D.D., Margolis, D.M., Delaney, M., Greene, W.C., Hazuda, D., and Pomerantz, R.J. (2009). The challenge of finding a cure for HIV infection. Science (80-. ). 323, 1304-1307.

Rivas, E., Clements, J., and Eddy, S.R. (2016). A statistical test for conserved RNA structure shows lack of evidence for structure in IncRNAs. Nat. Methods 14, 45-48.

Roder, K., Stirnemann, G., Wales, J., and Pasquali, S. (2019). Structural transitions in the RNA 7SK 5 hairpin and their effect on HEXIM binding. Nucleic Acids Res. 48, 1-17.

Rodnina, M. V., Fischer, N., Maracci, C., and Stark, H. (2017). Ribosome dynamics during decoding. Philos. Trans. R. Soc. B Biol. Sci. 372.

Roth, A., and Breaker, R.R. (2009). The structural and functional diversity of metabolite-binding riboswitches. Annu. Rev. Biochem. 78, 305-334.

Sengupta, A., Rice, G.M., and Weeks, K.M. (2019). Single-molecule correlated chemical probing reveals large-scale structural communication in the ribosome and the mechanism of the antibiotic spectinomycin in living cells. PLoS Biol. 17, 1-19.

Sharp, P.A. (2009). The Centrality of RNA. Cell 136, 577-580.

Shaw, L.M., Blanchard, A., Chen, Q., An, X., Davies, P., Tötemeyer, S., Zhu, Y.G., and Stekel, D.J. (2019). DirtyGenes: testing for significant changes in gene or bacterial population compositions from a small number of samples. Sci. Rep. 9, 1-10.

Siegfried, N. a, Busan, S., Rice, G.M., Nelson, J. a E., and Weeks, K.M. (2014). RNA motif discovery by SHAPE and mutational profiling (SHAPE-MaP). Nat. Methods 11, 959-965.

Sithole, N., Williams, C.A., Abbink, T.E.M., and Lever, A.M.L. (2020). DDX5 potentiates HIV-1 transcription as a co-factor of Tat. Retrovirology 17, 1-16.

Smola, M.J., Rice, G.M., Busan, S., Siegfried, N.A., and Weeks, K.M. (2015a). Selective 2'hydroxyl acylation analyzed by primer extension and mutational profiling (SHAPE-MaP) for direct, versatile and accurate RNA structure analysis. Nat. Protoc. 10, 1643-1669.

Smola, M.J., Calabrese, J.M., and Weeks, K.M. (2015b). Detection of RNA-Protein Interactions in Living Cells with SHAPE. Biochemistry 54, 6867-6875.

Studniarek, C., Tellier, M., Martin, P.G.P., Murphy, S., Kiss, T., and Egloff, S. (2021). The 7SK/P-TEFb snRNP controls ultraviolet radiation-induced transcriptional reprogramming. Cell Rep. 35.

Tan, J.L., Fogley, R.D., Flynn, R.A., Ablain, J., Yang, S., Saint-André, V., Fan, Z.P., Do, B.T., Laga, A.C., Fujinaga, K., et al. (2016). Stress from Nucleotide Depletion Activates the Transcriptional Regulator HEXIM1 to Suppress Melanoma. Mol. Cell 62, 34-46.

Tian, S., Kladwang, W., and Das, R. (2018). Allosteric Mechanism of the V. vulnificus Adenine Riboswitch Resolved by Four-dimensional Chemical Mapping. Elife 1-36.

Tomezsko, P.J., Corbin, V.D.A., Gupta, P., Swaminathan, H., Glasgow, M., Persad, S., 
Edwards, M.D., Mcintosh, L., Papenfuss, A.T., Emery, A., et al. (2020). Determination of RNA structural diversity and its role in HIV-1 RNA splicing. Nature 582, 438-442.

Tyagi, M., Pearson, R.J., and Karn, J. (2010). Establishment of HIV Latency in Primary CD4 + Cells Is due to Epigenetic Transcriptional Silencing and P-TEFb Restriction . J. Virol. 84, 64256437.

Wang, P.Y., Sexton, A.N., Culligan, W.J., and Simon, M.D. (2019). Carbodiimide reagents for the chemical probing of RNA structure in cells. Rna 25, 135-146.

Warhaut, S., Mertinkus, K.R., Hollthaler, P., Furtig, B., Heilemann, M., Hengesbach, M., and Schwalbe, H. (2017). Ligand-modulated folding of the full-length adenine riboswitch probed by NMR and single-molecule FRET spectroscopy. Nucleic Acids Res. 45, 5512-5522.

Wassarman, D.A., and Steitz, J.A. (1991). Structural analyses of the 7SK ribonucleoprotein (RNP), the most abundant human small RNP of unknown function. Mol. Cell. Biol. 11, 34323445.

Weeks, K.M. (2021). SHAPE Directed Discovery of New Functions in Large RNAs. Acc. Chem. Res. 54 (10), 2502-2517.

Wilkinson, M.E., Charenton, C., and Nagai, K. (2020). RNA Splicing by the Spliceosome. Annu. Rev. Biochem. 89, 359-388.

Yang, W., Wang, Y., Lai, A., Clark, C.G., Corte, J.R., Fang, T., Gilligan, P.J., Jeon, Y., Pabbisetty, K.B., Rampulla, R.A., et al. (2020). Discovery of a High Affinity, Orally Bioavailable Macrocyclic FXIa Inhibitor with Antithrombotic Activity in Preclinical Species. J. Med. Chem. 63, 7226-7242.

Yang, Y., Eichhorn, C.D., Wang, Y., Cascio, D., and Feigon, J. (2019). Structural basis of 7SK RNA 5'- $\gamma$-phosphate methylation and retention by MePCE. Nat. Chem. Biol. 15, 132-140.

Yang, Z., Zhu, Q., Luo, K., and Zhou, Q. (2001). The 7SK small nuclear RNA inhibits the CDK9/cyclin T1 kinase to control transcription. Nature 414, 317-322.

Yazbeck, A.M., Tout, K.R., and Stadler, P.F. (2018). Detailed secondary structure models of invertebrate 7SK RNAs. RNA Biol. 15, 158-164.

Zhang, J., and Ferré-D'Amaré, A.R. (2014). Dramatic improvement of crystals of large RNAs by cation replacement and dehydration. Structure 22, 1363-1371. 


\section{Figure Legends}

Figure 1: Maximum likelihood deconvolution enables thermodynamically rigorous analysis of RNA conformational ensembles. (A) Schematic of ML ensemble deconvolution. RNAs with multiple structures generate distinctive chemical modification patterns in single-molecule MaP data. For an RNA sampling multiple states, a (conventional) averaged, per-nucleotide reactivity profile may not be representative of any of the underlying structure states. $\mathrm{ML}$ analysis reveals both the individual reactivity profiles and populations of each ensemble state. (B) Averaged and deconvoluted DMS-MaP data for the ON and OFF states of the add adenine riboswitch. High, medium and low DMS reactivities are shown in red, yellow and black, respectively. Major reactivity differences between the ON and OFF states are shaded. (C) Deconvoluted structures of the add riboswitch. (left) Deconvoluted MaP data for the add riboswitch in the absence of ligand, superimposed on the NMR-defined (Reining et al., 2013) ON and OFF ensemble states. Muton (Reining et al., 2013) and Mutoff mutants are also illustrated. (right) State-specific pairing probabilities for the add ON and OFF states computed from deconvoluted reactivity profiles at 0 $\mu \mathrm{M}$ adenine. (D) Population of the $\mathrm{ON}$ state as a function of adenine concentration for native and mutant sequences. Replicates are shown in closed and open symbols. (E) Reactivities for the ON and OFF states as a function of adenine concentration. Regions that undergo adeninedependent protection are emphasized with gray shading. Complete ensembles at each concentration are shown in Fig. S3 and S4. The add RNA is numbered per prior convention (Reining et al., 2013).

Figure 2: Direct DANCE-MaP detection of state-specific RNA base pairs and tertiary interactions. (A) DMS induces correlated disruptions of base pairing and tertiary interactions, measurable by MaP. Following ML deconvolution of per-nucleotide reactivity profiles, individual reads are assigned to distinct states. PAIR and RING correlation analyses are then used to directly detect base pairing and through-space tertiary interactions, respectively. (B,C) PAIR and RING analyses of composite (left) and deconvoluted (right) adenine riboswitch datasets, measured at 0 and $100 \mu \mathrm{M}$ adenine. PAIRs are shown at top, superimposed on the modeled secondary structure state (light gray). High and moderate confidence PAIRs (denoted principal and minor in (Mustoe et al., 2019)) are dark and light blue, respectively. Arrows denote structure-specific PAIRs only observed in deconvoluted data. RINGs are shown at bottom, colored according to statistical significance. PAIR and RING correlations are also drawn on conventional secondary structure diagrams (right). RINGs measured in the ON state are 
superimposed on the crystal structure of the adenine riboswitch aptamer domain (far right; PDB: 4tzx (Zhang and Ferré-D’Amaré, 2014)).

Figure 3: The 7SK RNA intrinsically codes for a large-scale structural switch. (A) Averaged and DANCE-deconvoluted reactivity profiles for 7SK RNA in cells. Major differences between states $A$ and $B$ are highlighted with gray shading. State $H$ (heterogenous) has high reactivity throughout. Population averages and standard deviations are computed over 10 replicates (Table S2). Stem loop (SL) structural landmarks are indicated at bottom. (B) Comparison of state $A$ and $B$ per-nucleotide reactivities for in-cell and cell-free 7SK. Person's R is shown. (C) In-cell and cell-free structural models for states $A$ and $B$. Modeled base pairing probabilities (top) and directly measured PAIRs (bottom) are shown as arcs. (D) Consensus secondary structure models, shown in individual base pair format. Per-nucleotide reactivities are colored as per panel A. RINGs observed for state B are shown with orange-red lines, consistent with through-space structural communication and tertiary interactions. Measured PAIRs that directly support either state A or B are boxed in green; PAIRs observed in one versus both in-cell and cell-free datasets are shown with blue and magenta lines, respectively. Replicate data and state A and cell-free RINGs are shown in Figure S6.

Figure 4: Assessment and validation of 7 SK states $A$ and $B$ by mutational analysis. (A) RNA mutants. Mutations are shown superimposed on consensus state $A$ and $B$ structural models. (B) Summary of designed structural impact for each mutant. (C) Ensemble distribution observed for the native sequence RNA and each mutant, produced as in vitro transcripts. Structural models are provided in Fig. S9.

Figure 5: The 7SK equilibrium is regulated by cell type and growth conditions. (A) 7SK structural ensemble in proliferating RPE-1 cells resolved by DANCE-MaP. Structure models are shown as in Figure 3C. Pearson's R, comparing RPE-1 and Jurkat cell reactivities, are shown. (B) 7SK ensemble populations for Jurkat cells and proliferating and quiescent RPE-1 cells. Comparisons between individual state populations were evaluated using a two-sided MannWhitney $U$ test. Inset, population data shown in complete distribution format. Comparisons between complete ensembles were performed using a Dirichlet likelihood ratio test (Shaw et al., 2019). $N=10,7$, and 3 for Jurkat, proliferating RPE-1, and quiescent RPE-1 cells, respectively. ${ }^{*}, p<0.05 ;{ }^{* *}, p<0.01 ;{ }^{* * *}, p<0.001 ;{ }^{* * *}, p<0.0001$ (C) Shift in 7SK equilibrium upon flavopiridol treatment in Jurkat and RPE-1 cells. Cells were treated with vehicle (DMSO, $0.01 \%$ ) or $1 \mu \mathrm{M}$ 
flavopiridol for $1 \mathrm{hr}(\mathrm{N}=2$, all experiments).

Figure 6: Stabilization of 7SK state B induces transcription. (A) ASO-B binding site shown superimposed on secondary structure models of 7SK states A and B. (B) Engagement of ASO$B$ on the 7SK RNA observed as a complete reduction of per-nucleotide reactivity at the ASO binding site. MM-B: control ASO containing 5 central mismatches. (C) DANCE deconvolution reveals ASO-B-induced states all correspond to B/B-like states. (D/E) 7SK and HEXIM1 RNA levels measured by RT-qPCR. HEK293 cells were treated for 24 hours with oligos prior to total RNA extraction and quantification. Data normalized to the control gene TBP, in three triplicate experiments. (D) GAP-B induces a decrease in 7SK expression (top) and a dose-dependent increase in HEXIM1 expression (bottom). $\mathrm{N}=3$ for 200 nM GAP-B; $\mathrm{N}=6$ for all other measurements. (E) 7SK expression shows (top) no change when treated with ASO-B, MM-B, or JQ1. HEXIM1 shows (bottom) significant increase in expression for ASO-B compared to MM-B. $N=9$ for all measurements. Significance determined using Welch's t-test ${ }^{*}, p<0.05 ;{ }^{* *}, p<0.01$; $\left.{ }^{* * *}, p<0.001 ;{ }^{* * * *}, p<0.0001\right)$.

Figure 7: The 7SK ensemble as a dual-function signal integrator. States $A, B, H$ are shown as schematic secondary structures. Compact core corresponds to region of dense RINGs. Annotations show binding sites of core factors (P-TEFb, HEXIM1/2, MePCE and LaRP7), approximate region bound by helicase and other release factors (blue outline), and postulated sites of pro-transcription factors. 
bioRxiv preprint doi: https://doi.org/10.1101/2021.09.16.460563; this version posted September 16, 2021. The copyright holder for this preprint

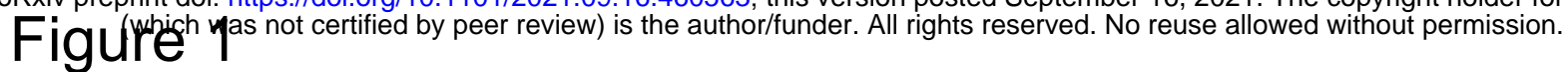
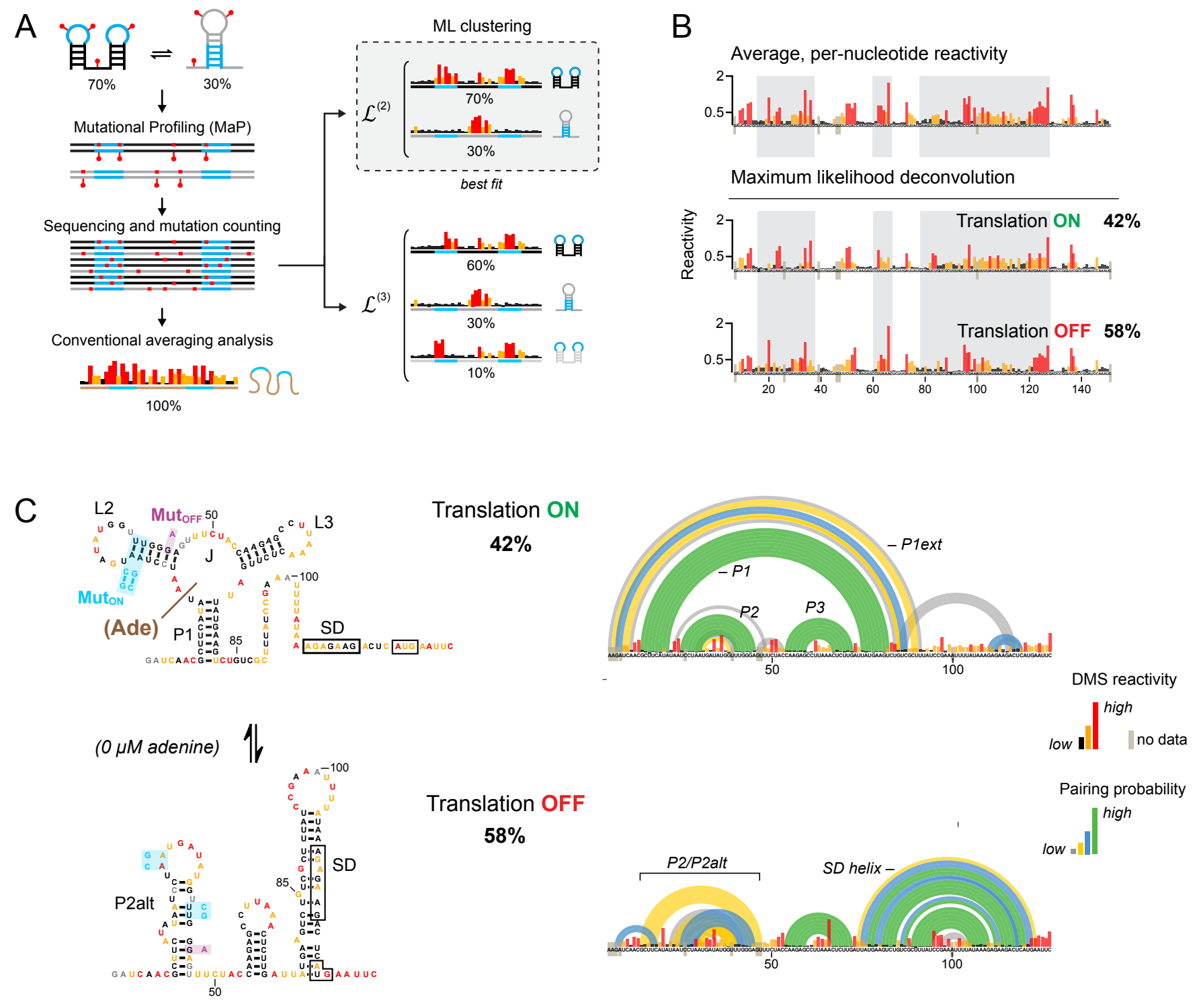

D

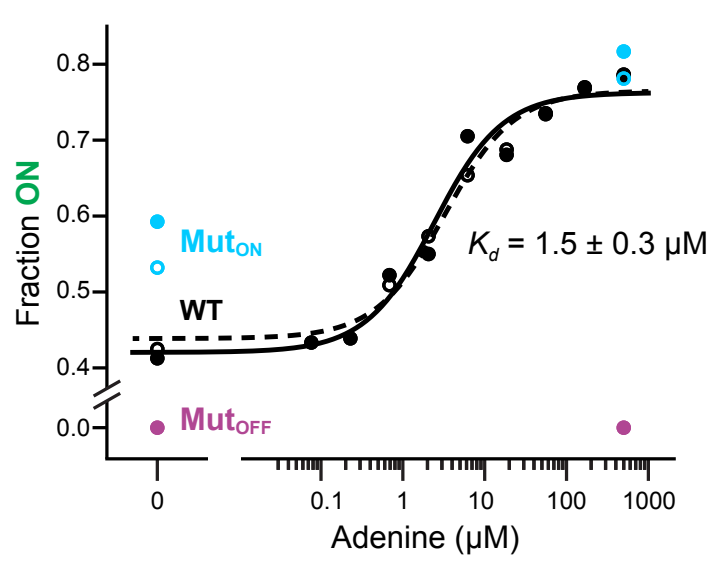

$\mathrm{E}$

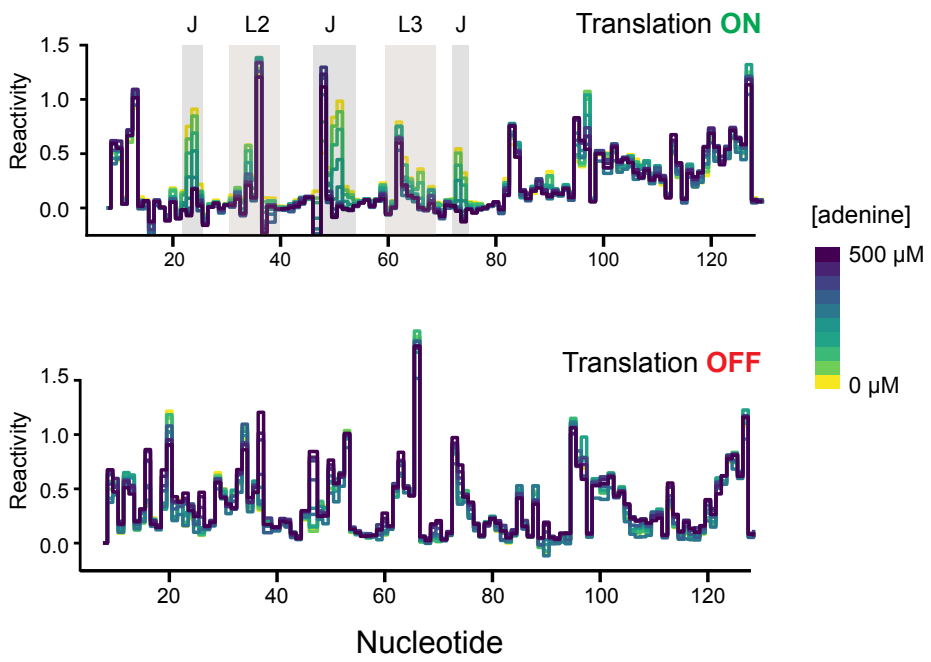


bioRxiv preprint doi: https://doi.org/10.1101/2021.09.16.460563; this version posted September 16, 2021. The copyright holder for this preprint Figuren mas not certified by peer review) is the author/funder. All rights reserved. No reuse allowed without permission.
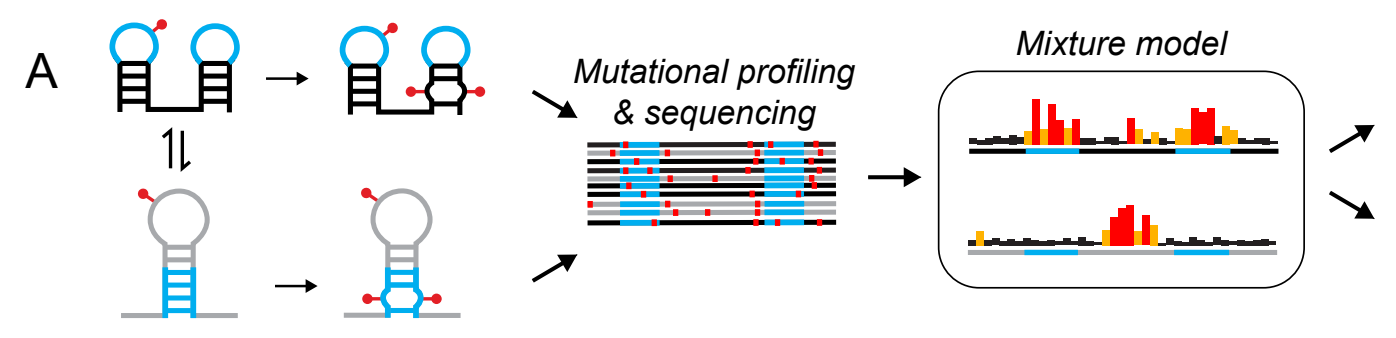

PAIRs -

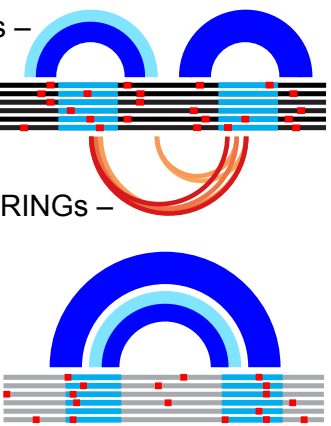

Invert ML framework to assign individual reads and compute through-space PAIR and RING interactions for each state

\section{Composite, average}

B

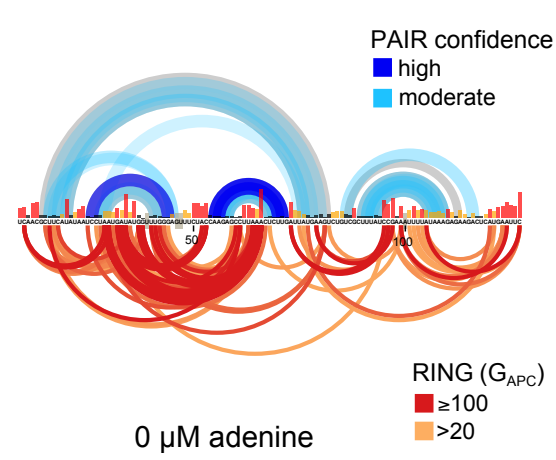

C

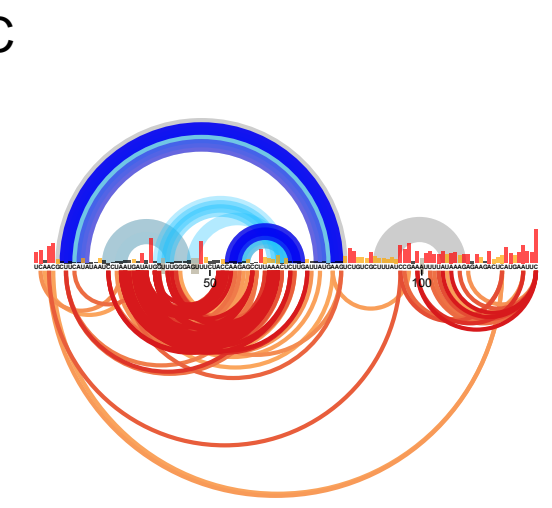

$100 \mu \mathrm{M}$ adenine
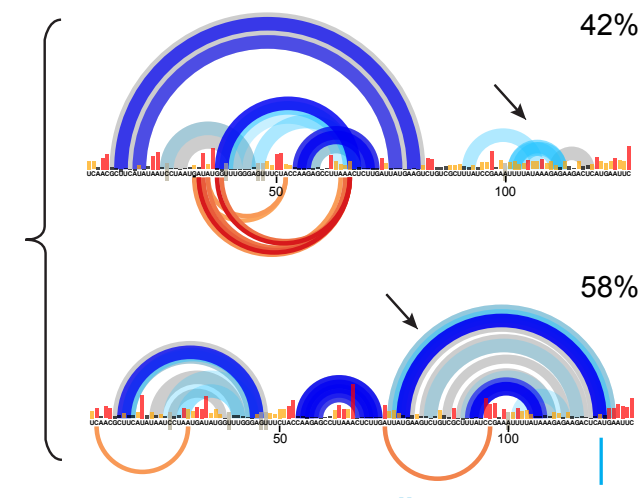

direct measurement of base pairing (PAIRs) in each state

direct measurement of through-space (RING) interactions
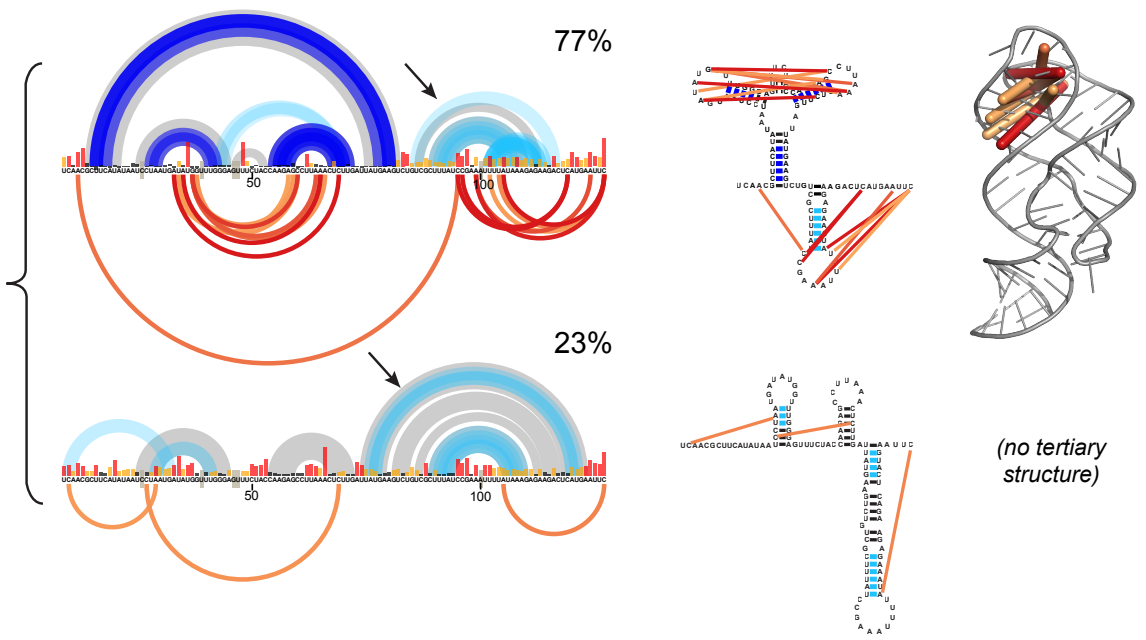

(no tertiary structure) 
bioRxiv preprint doi: https://doi.org/10.1101/2021.09.16.460563; this version posted September 16, 2021. The copyright holder for this preprint

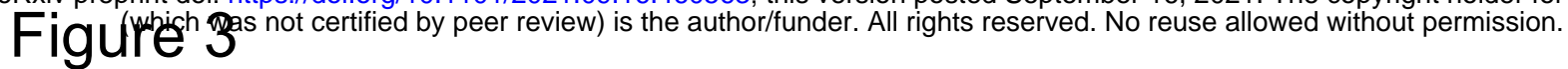

A
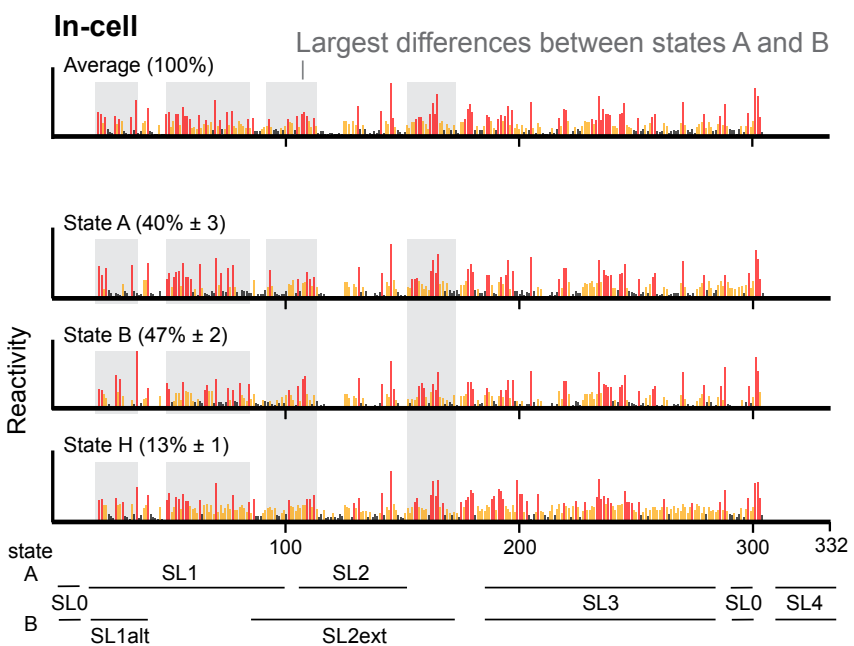

C

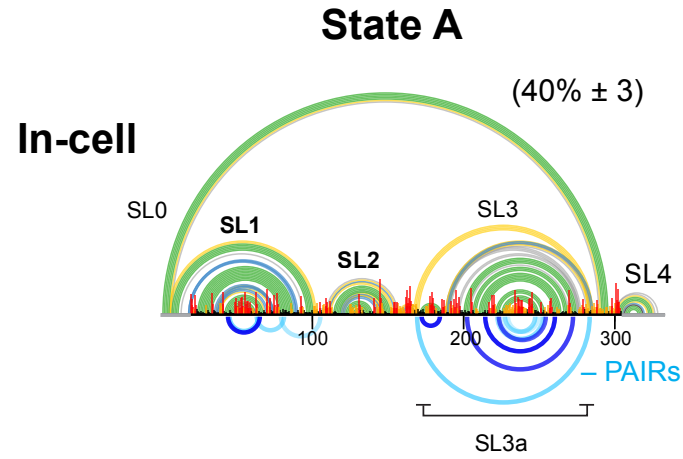

$(43 \% \pm 8)$

\section{Cell-free}

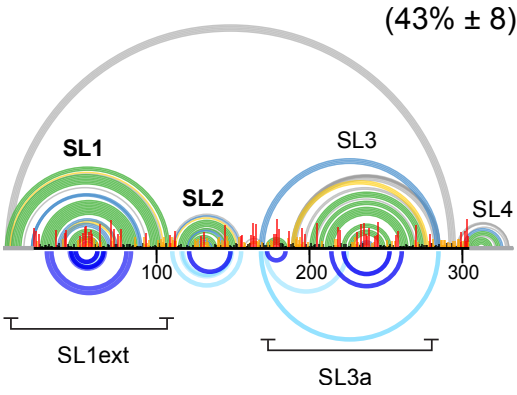

D Consensus models

SL2

SL1 $1^{\cup \sim A_{c i g}}$

PAIRs observed both

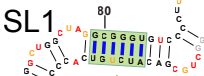

PAIRs unique to

either state A or B

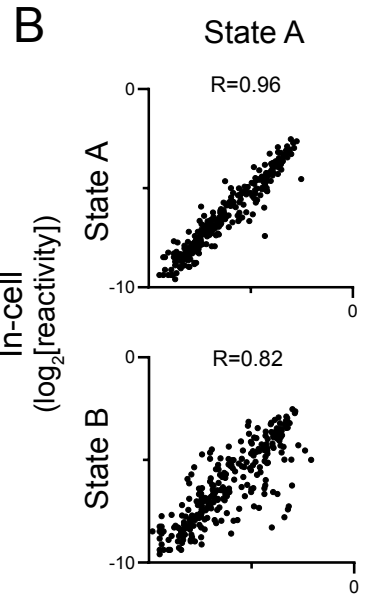

State B
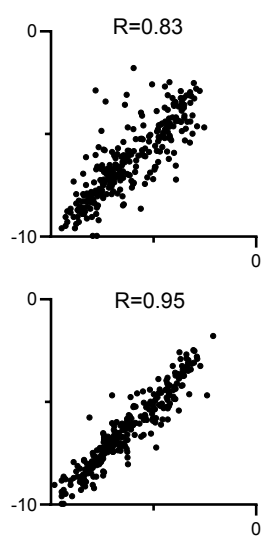

Cell-free $\left(\log _{2}\right.$ [reactivity])

State B
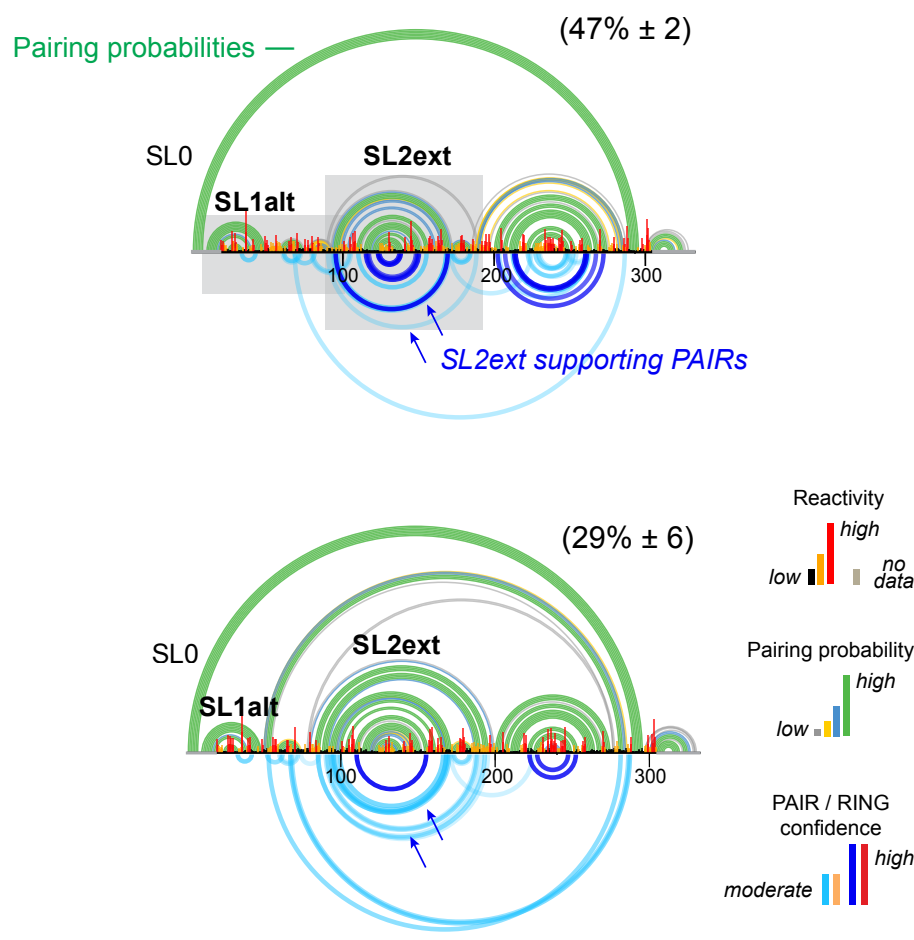

PAIR / RING moderate || 
bioRxiv preprint doi: https://doi.org/10.1101/2021.09.16.460563; this version posted September 16, 2021. The copyright holder for this preprint Figulyejeh yas not certified by peer review) is the author/funder. All rights reserved. No reuse allowed without permission.

A

State A

State B
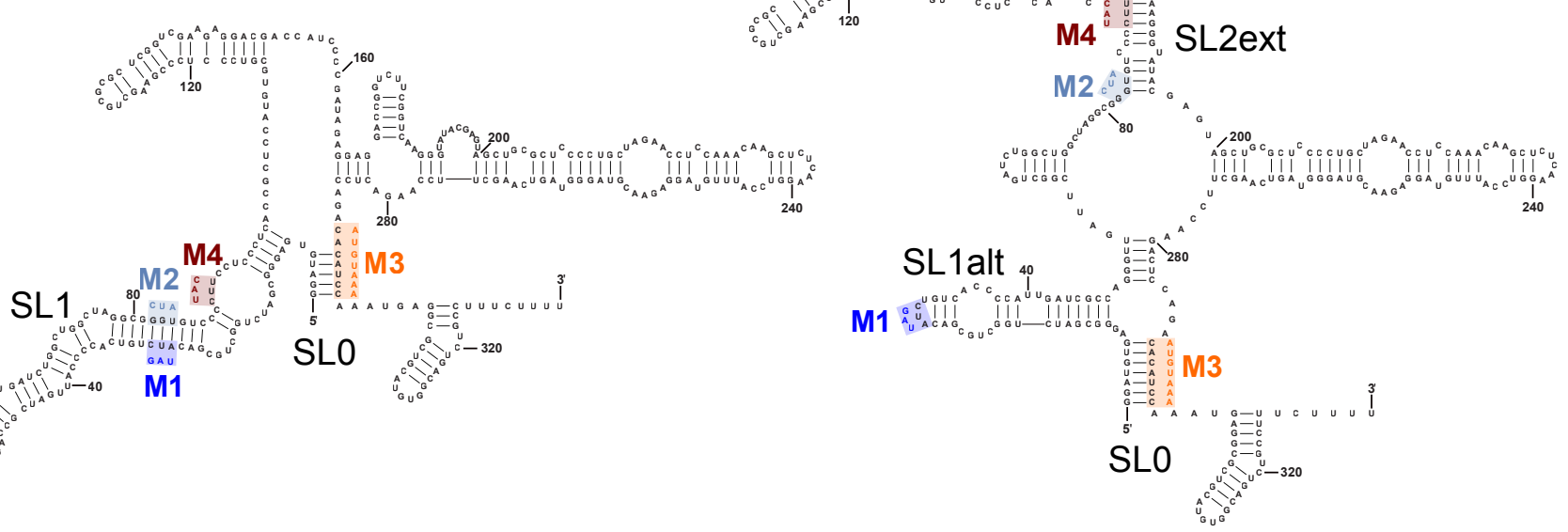

\begin{tabular}{|c|c|c|}
\hline Mutant & Structure change & Expected Result \\
\hline M1 & Disrupt SL1 & Favor B \\
\hline M1+M2 & Restore SL1 & Restore equilibrium \\
\hline M3 & Disrupt SL0 & No impact \\
\hline M4 & Disrupt SL2alt & Favor A \\
\hline
\end{tabular}

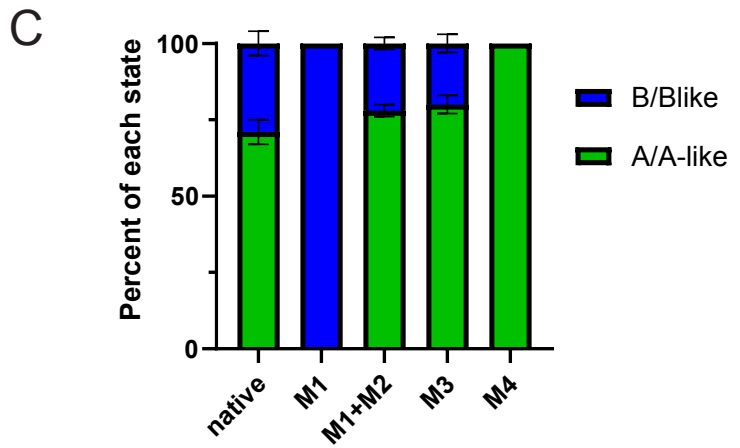




\section{Figure 5}

A State A

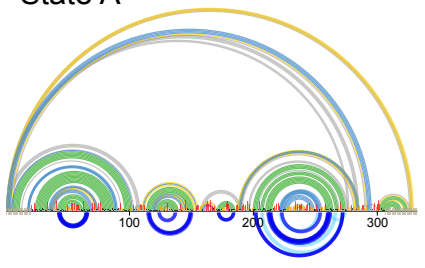

$$
47 \% \pm 2
$$

$\mathrm{R}_{\text {Jurkat }}=0.99$

B

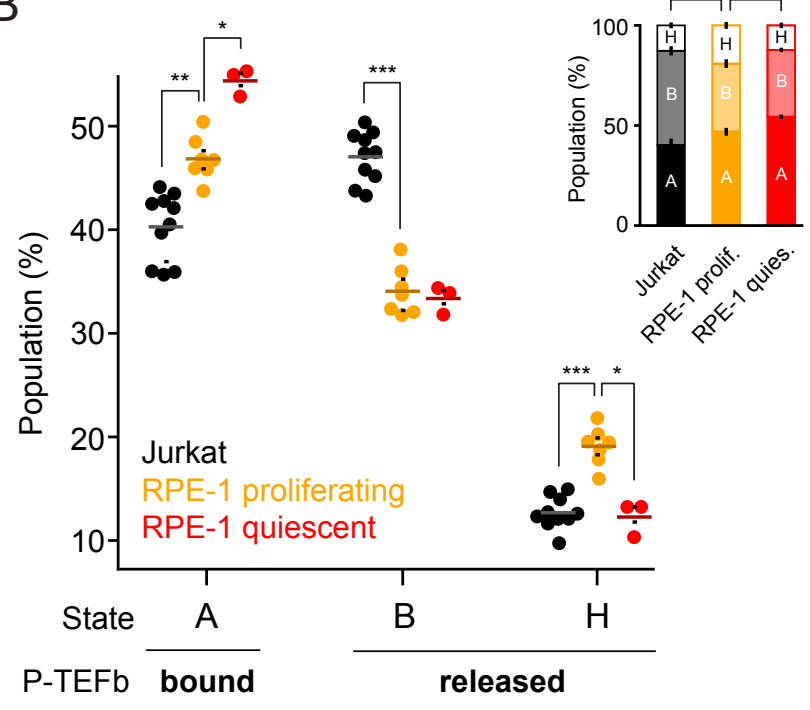

State B

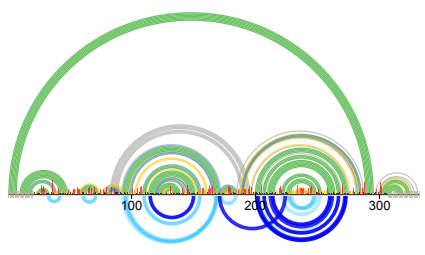

$$
34 \% \pm 2
$$$$
\mathrm{R}_{\text {Jurkat }}=0.99
$$

State $\mathrm{H}$

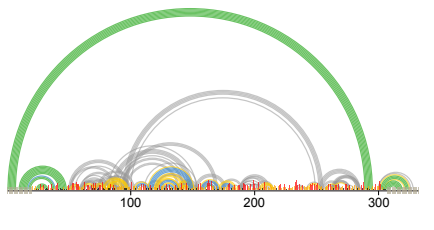

$$
\begin{gathered}
19 \% \pm 2 \\
\mathrm{R}_{\text {Jurkat }}=0.92
\end{gathered}
$$

C

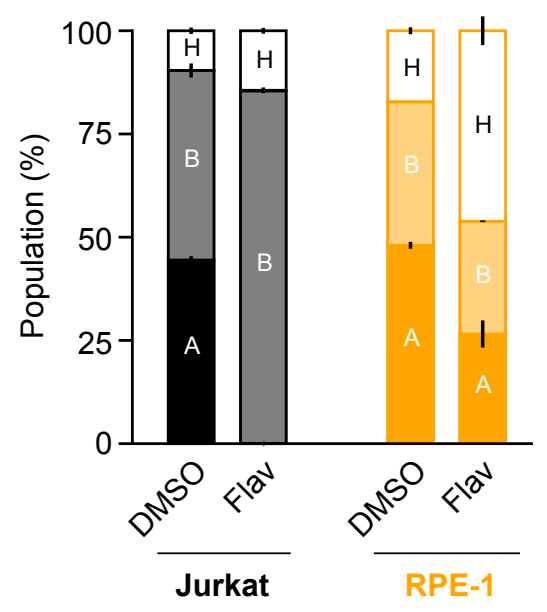


bioRxiv preprint doi: https://doi.org/10.1101/2021.09.16.460563; this version posted September 16, 2021. The copyright holder for this preprint Figulwhich as not certified by peer review) is the author/funder. All rights reserved. No reuse allowed without permission.
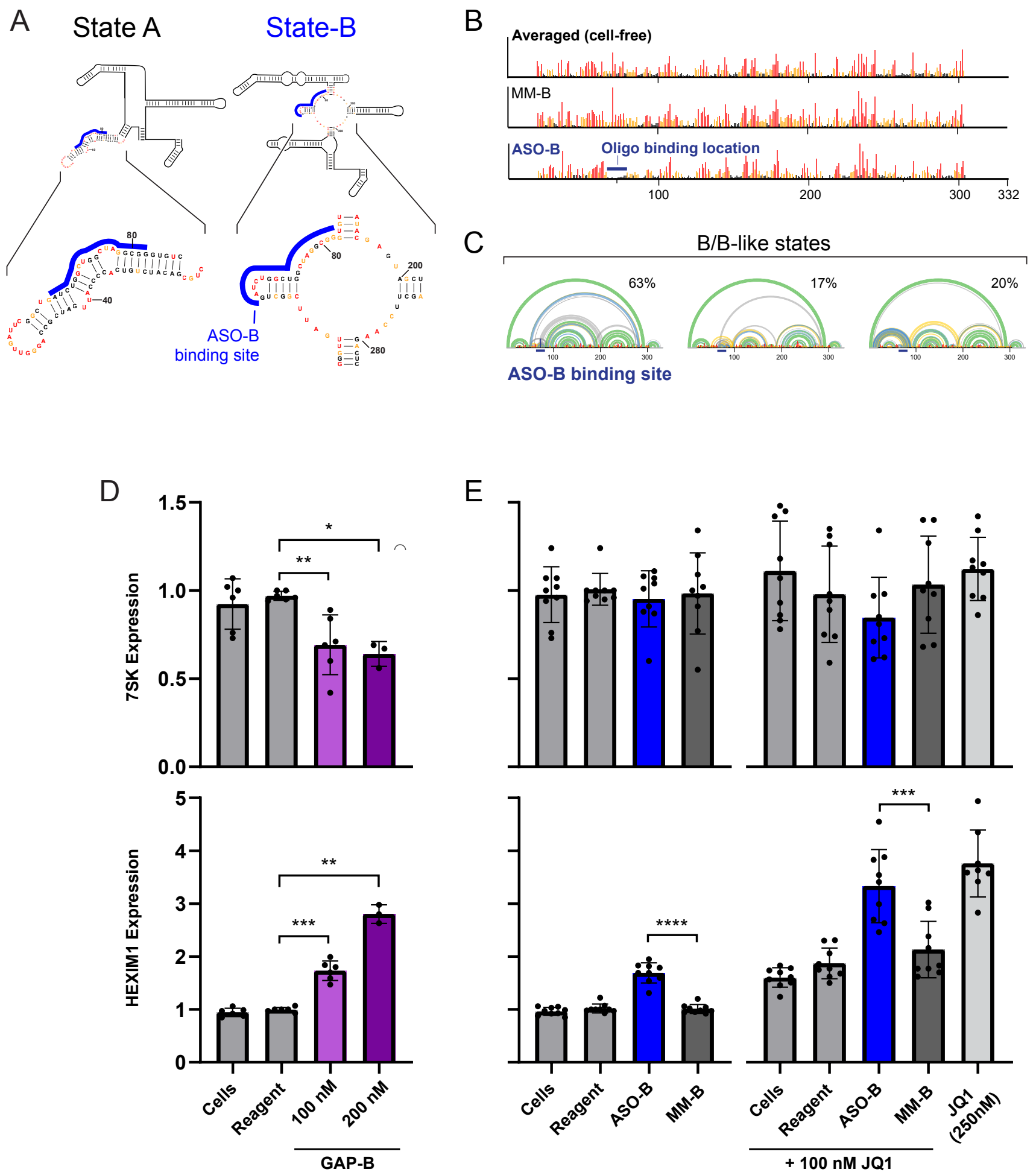
bioRxiv preprint doi: https://doi.org/10.1101/2021.09.16.460563; this version posted September 16, 2021. The copyright holder for this preprint (which was not certified by peer review) is the author/funder. All rights reserved. No reuse allowed without permission.

\section{Figure 7}

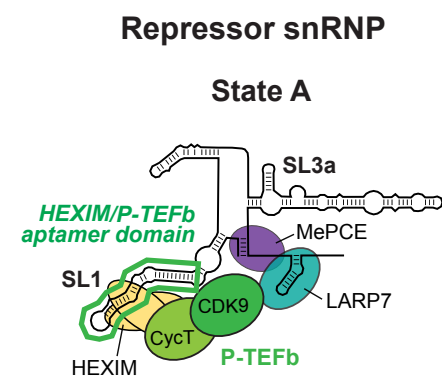

Release factors effect P-TEFb release via allosteric structural switch

Pro-transcription snRNP

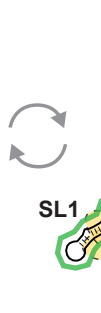
State A

State B

State B/H

Release factor
binding sites
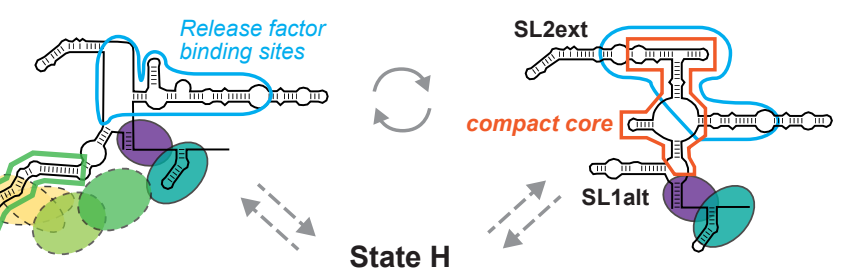

State $\mathbf{H}$
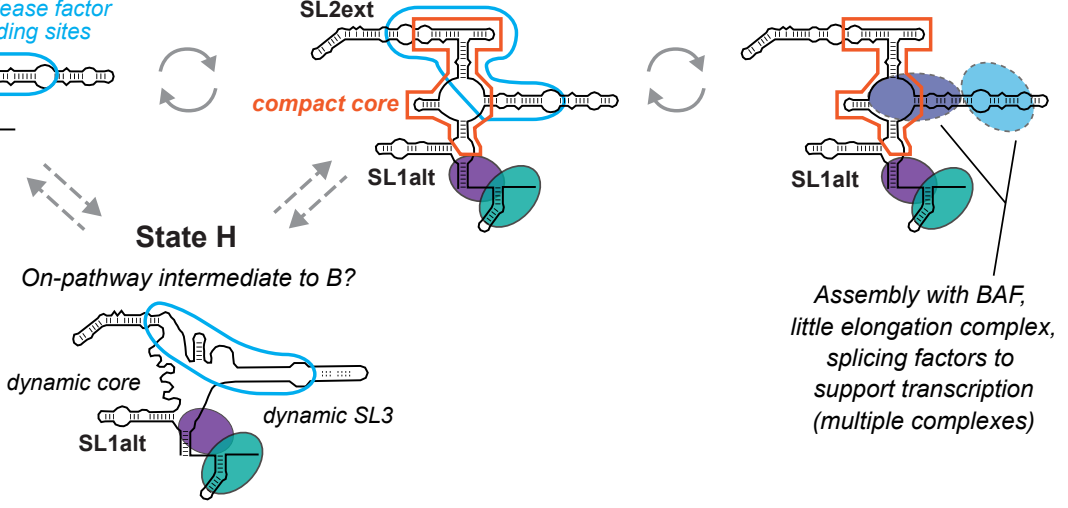

Assembly with $B A F$,

little elongation complex, splicing factors to support transcription (multiple complexes) 